\title{
Long time motion of NLS solitary waves in a confining potential
}

\section{Journal Article}

\section{Author(s):}

Jonsson, B. Lars G.; Fröhlich, Jürg; Gustafson, Stephen; Sigal, Israel Michael

Publication date:

2006-06

Permanent link:

https://doi.org/10.3929/ethz-b-000022838

Rights / license:

In Copyright - Non-Commercial Use Permitted

Originally published in:

Annales Henri Poincaré 7(4), https://doi.org/10.1007/s00023-006-0263-y 


\title{
Long Time Motion of NLS Solitary Waves in a Confining Potential
}

\author{
B. Lars G. Jonsson*, Jürg Fröhlich, Stephen Gustafson ${ }^{\dagger}$ \\ and Israel Michael Sigal ${ }^{\ddagger}$
}

\begin{abstract}
We study the motion of solitary-wave solutions of a family of focusing generalized nonlinear Schrödinger equations with a confining, slowly varying external potential, $V(x)$.

A Lyapunov-Schmidt decomposition of the solution combined with energy estimates allows us to control the motion of the solitary wave over a long, but finite, time interval.

We show that the center of mass of the solitary wave follows a trajectory close to that of a Newtonian point particle in the external potential $V(x)$ over a long time interval.
\end{abstract}

\section{Introduction}

We consider a family of generalized nonlinear Schrödinger (NLS), and Hartree equations. For a large class of nonlinearities these equations have solitary wave solutions, and, in this paper, we study the effective dynamics of such solitary waves. The equations have the form:

$$
\mathrm{i} \partial_{t} \psi(x, t)=-\Delta \psi(x, t)+V(x) \psi(x, t)-f(\psi)(x, t),
$$

where $t \in \mathbb{R}$ is time, $x \in \mathbb{R}^{d}$ denotes a point in physical space, $\psi: \mathbb{R}^{d} \times \mathbb{R} \mapsto \mathbb{C}$ is a (one-particle) wave function, $V$ is the external potential, which is a real-valued, confining, and slowly varying function on $\mathbb{R}^{d}$, and $f(\psi)$ describes a nonlinear selfinteraction with the properties that $f(\psi)$ is "differentiable" in $\psi, f(0)=0$, and $f(\bar{\psi})=\overline{f(\psi)}$. Precise assumptions on $V$ and $f$ are formulated in Section 2 .

\footnotetext{
* Supported by the Swiss National Foundation under NF-Project 20-105493 and by 'The Year of PDE' at The Fields Institute.

$\dagger$ Supported by NSERC 22R80976.

$\ddagger$ Supported by NSERC under Grant NA7901.
} 
The family of nonlinearities of interest to us includes local nonlinearities, such as

$$
f(\psi)=\lambda|\psi|^{s} \psi, \quad 0<s<\frac{4}{d}, \lambda>0,
$$

and Hartree nonlinearities

$$
f(\psi)=\lambda\left(\Phi *|\psi|^{2}\right) \psi, \quad \lambda>0,
$$

where the (two-body) potential $\Phi$ is real-valued, of positive type, continuous, spherically symmetric, and tends to 0 as $|x| \rightarrow \infty$. Here $\Phi * g:=\int \Phi(x-y) g(y) \mathrm{d}^{d} y$ denotes convolution. Such equations are encountered in the theory of Bose gases (BEC), in nonlinear optics, in the theory of water waves and in other areas of physics.

It is well known that for a large class of nonlinarities Eq. (1.1) has solitary wave solutions when $V \equiv 0$. Let $\eta_{\mu} \in \mathrm{L}^{2}$ be a spherically symmetric, positive solution of the nonlinear eigenvalue problem

$$
-\Delta \eta+\mu \eta-f(\eta)=0 .
$$

The function $\eta_{\mu}$ is called a "solitary wave profile". Among the solitary wave solutions of (1.1) are Galilei transformations of $\eta_{\mu}$,

$$
\psi_{\text {sol }}:=\mathcal{S}_{a(t) p(t) \gamma(t)} \eta_{\mu(t)},
$$

where $\mathcal{S}_{a p \gamma}$ is defined by

$$
\left(\mathcal{S}_{a p \gamma} \psi\right)(x):=\mathrm{e}^{\mathrm{i} p \cdot(x-a)+\mathrm{i} \gamma} \psi(x-a) .
$$

Let $\sigma:=\{a, p, \gamma, \mu\}$, where $\mu$ is as in Eq. (1.4). For $\psi_{\text {sol }}$ to be a solution to (1.1) with $V \equiv 0$ the modulation parameters, $\sigma$, must satisfy the equations of motion

$$
a(t)=2 p t+a, \quad p(t)=p, \quad \gamma(t)=\mu t+p^{2} t+\gamma, \quad \mu(t)=\mu
$$

with $\gamma \in \mathbb{S}^{1}, a, p \in \mathbb{R}^{d}, \mu \in \mathbb{R}_{+}$. In other words, if $\sigma$ satisfy (1.7), then

$$
\psi_{\text {sol }}(x, t)=\left(\mathcal{S}_{a(t) p(t) \gamma(t)} \eta_{\mu(t)}\right)(x)
$$

solves Eq. (1.1) with $V \equiv 0$. Let the soliton manifold, $\mathrm{M}_{\mathrm{s}}$ be defined by

$$
\mathrm{M}_{\mathrm{s}}:=\left\{\mathcal{S}_{a p \gamma} \eta_{\mu}:\{a, p, \gamma, \mu\} \in \mathbb{R}^{d} \times \mathbb{R}^{d} \times \mathbb{S}^{1} \times I\right\},
$$

where $I$ is a bounded interval in $\mathbb{R}_{+}$.

Solutions to (1.4) behave roughly like $\mathrm{e}^{-\sqrt{\mu}|x|}$, as $|x| \rightarrow \infty$. So $\mu^{-1 / 2}$ is a length scale that indicates the "size" of the solitary wave.

We consider the Cauchy problem for Eq. (1.1), with initial condition $\psi_{0}$ in a weighted Sobolev space. For Hartree nonlinearities, global wellposedness is known [28]. For local nonlinearities, the situation is more delicate; see Condition 1 and Remark 2.1 in Section 2. Let the initial condition $\psi_{0}$ be "close" to $\mathrm{M}_{\mathrm{s}}$. Then, we will show, the corresponding solution $\psi$ will remain "close" to $\mathrm{M}_{\mathrm{s}}$, over a long time interval. A certain "symplectically orthogonal" projection of $\psi$ onto $\mathrm{M}_{\mathrm{S}}$ is then well defined and traces out a unique curve on $\mathrm{M}_{\mathrm{s}}$. We denote this curve by $\eta_{\sigma(t)}$, see Figure 1.1. 


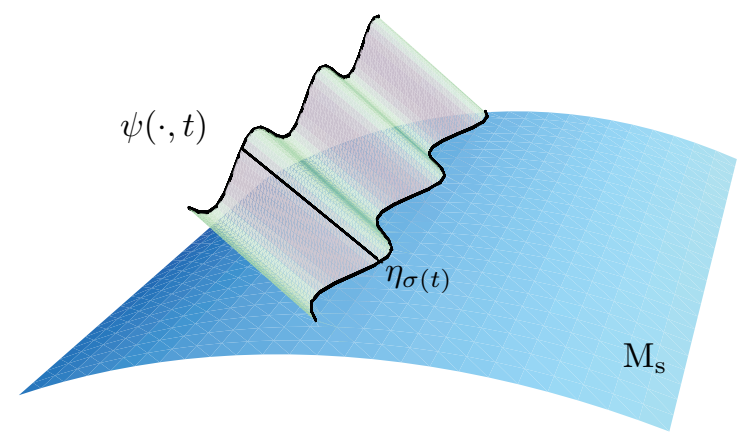

FigurE 1.1. The trajectory $\psi(\cdot, t)$ over the soliton Manifold $\mathrm{M}_{\mathrm{s}}$.

An essential part of this paper is to determine the leading order behavior of $\sigma(t)=\{a(t), p(t), \gamma(t), \mu(t)\}$ and to estimate error terms. To this end, let $W$ be a smooth, positive, polynomially bounded function, and define

$$
V(x)=W\left(\epsilon_{V} x\right)
$$

where $\epsilon_{V}$ is a small parameter. Furthermore, let $\psi_{0}$ be an initial condition " $\epsilon_{0}$ close" to $\eta_{\sigma_{0}} \in \mathrm{M}_{\mathrm{s}}$, for some $\sigma_{0}$. Roughly speaking, this initial condition has length scale $1 / \sqrt{\mu}_{0}$. We will consider external potentials, $V$, as in (1.10), for a scaling parameter $\epsilon_{V}$ satisfying

$$
\epsilon_{V} \ll \sqrt{\mu_{0}}
$$

i.e., we assume that the external potential varies very little over the length scale of $\psi_{0}$. For simplicity, we choose $\mu_{0}=\mathcal{O}(1)$ and $\epsilon_{V} \ll 1$, at the price of re-scaling the nonlinearity.

We decompose the solution $\psi$ of (1.1) into a part which is a solitary wave and a small part, a "perturbation", $w$. That is, we write $\psi$ as

$$
\psi=\mathcal{S}_{a p \gamma}\left(\eta_{\mu}+w\right) .
$$

This does not define a unique decomposition, unless $2 d+2$ additional conditions are imposed. These conditions say that the perturbation $w$ is 'symplectically orthogonal' to the soliton manifold $\mathrm{M}_{\mathrm{s}}$.

One of our main steps is a derivation of differential equations in time for the modulation parameters, $\sigma$, which depend on the external potential. These equations appear naturally when one projects solutions of (1.1) onto the soliton manifold. To control the motions of $\sigma$ and $w$, we make use of conserved quantities: the energy

$$
\mathcal{H}_{V}(\psi):=\frac{1}{2} \int\left(|\nabla \psi|^{2}+V|\psi|^{2}\right) \mathrm{d}^{d} x-F(\psi)
$$


where $F^{\prime}(\psi)=f(\psi)$ (this is a variational derivative), the mass (or charge)

$$
\mathcal{N}(\psi):=\frac{1}{2} \int|\psi|^{2} \mathrm{~d}^{d} x
$$

and the "almost conserved" momentum

$$
\mathcal{P}(\psi):=\frac{1}{4} \int(\bar{\psi} \nabla \psi-\psi \nabla \bar{\psi}) \mathrm{d}^{d} x .
$$

To achieve control over the perturbation $w$, we introduce a 'Lyapunov functional'

$$
\Lambda(\psi, t):=K_{\sigma}(\psi)-K_{\sigma}\left(\mathcal{S}_{a p \gamma} \eta_{\mu}\right),
$$

where $\sigma=\sigma(t)=\{a(t), p(t), \gamma(t), \mu(t)\}$, and where

$$
\begin{aligned}
K_{\sigma}(\psi):= & \mathcal{H}_{V}(\psi)+\left(p^{2}+\mu\right) \mathcal{N}(\psi)-2 p \cdot \mathcal{P}(\psi) \\
& -\frac{1}{2} \int(V(a)+\nabla V(a) \cdot(x-a))|\psi|^{2} \mathrm{~d}^{d} x,
\end{aligned}
$$

i.e., $K_{\sigma}$ is essentially a linear combination of the conserved and almost conserved quantities. Using the linear transformation $u:=\mathcal{S}_{a p \gamma}^{-1} \psi$, we change questions about the size of fluctuations around $\mathcal{S}_{a p \gamma} \eta_{\mu}$ to ones about the size of fluctuations around the solitary wave profile $\eta_{\mu(t)}$. In this "moving frame", the $K_{\sigma}(\psi)$ terms in the Lyapunov functional introduced above take the form

$$
K_{\sigma}\left(\mathcal{S}_{a p \gamma} u\right)=\mathcal{E}_{\mu}(u)+\frac{1}{2} \int \mathcal{R}_{V}|u|^{2} \mathrm{~d}^{d} x
$$

where

$$
\mathcal{R}_{V}(x):=V(x+a)-V(a)-\nabla V(a) \cdot(x-a)
$$

and

$$
\mathcal{E}_{\mu}(u):=\mathcal{H}_{V=0}(u)+\mu \mathcal{N}(u) .
$$

In the moving frame the Lyapunov functional depends on the parameters $\mu$ and $a$, but not on $p$ and $\gamma$. Furthermore, $\eta_{\mu}$ is a critical point of $\mathcal{E}_{\mu}\left(\eta_{\mu}\right)$, i.e., $\mathcal{E}_{\mu}^{\prime}\left(\eta_{\mu}\right)=0$. The change of frame discussed above simplifies the analysis leading to our main result.

Simply stated, our main theorem shows that, for initial conditions $\psi_{0} \epsilon_{0}$-close to $\mathrm{M}_{\mathrm{s}}$, the perturbation $w$ is of order $\epsilon:=\epsilon_{V}+\epsilon_{0}$, for all times smaller than $C \epsilon^{-1}$. Furthermore, the center of mass of the solitary wave, $a$, and the center of mass momentum $p$ satisfy the following equations

$$
\dot{a}=2 p+\mathcal{O}\left(\epsilon^{2}\right), \quad \dot{p}=-\nabla V(a)+\mathcal{O}\left(\epsilon^{2}\right) .
$$

The remaining modulation parameters $\mu$ and $\gamma$ satisfy

$$
\dot{\mu}=\mathcal{O}\left(\epsilon^{2}\right), \quad \dot{\gamma}=\mu-V(a)+p^{2}+\mathcal{O}\left(\epsilon^{2}\right) .
$$

A precise statement is found in the next section.

This is the first result of its type covering confining external potentials. Indeed, we can exploit the confining nature of the potential to obtain a stronger 
result than that of [17] (and that stated above) for a certain class of initial conditions which we now describe. Consider the classical Hamiltonian function:

$$
h(a, p):=\left(p^{2}+V(a)\right) / 2 .
$$

Given an initial condition $\psi_{0} \epsilon_{0}$-close to $\eta_{\sigma_{0}} \in \mathrm{M}_{\mathrm{s}}$, where $\sigma_{0}=\left\{a_{0}, p_{0}, \gamma_{0}, \mu_{0}\right\}$, we require the initial position $a_{0}$ and momentum $p_{0}$ to satisfy

$$
h\left(a_{0}, p_{0}\right)-\min _{a} h(a, 0) \leq \epsilon_{h},
$$

with $\epsilon_{V} \leq C \epsilon_{h} \leq 1$, for some constant $C$. For this class of initial conditions, our main result shows that the perturbation $w$ remains $\mathcal{O}(\epsilon)$ for longer times:

$$
t<\frac{C}{\epsilon_{V} \sqrt{\epsilon_{h}}+\epsilon^{2}} .
$$

This improvement is non-trivial. For example, it means that we can control the perturbation of a solitary wave which undergoes many oscillations near the bottom of a potential well.

One should contrast the effective soliton dynamics described in Eqns. (1.21) and (1.22) with the Ehrenfest equations for the NLS which hold - just like the conservation of energy - for energy-space solutions, under mild assumptions on the potential and the nonlinearity (see $[16,22,15,32,17])$.

Note that the decomposition (1.12) of the solution of Eqn. (1.1) is in the spirit of the modulation analysis, and Eqns. (1.21), (1.22) can be seen as modulation equations derived from imposing a 'biorthogonality' condition (equivalent to symplectic orthogonality in the present, Hamiltonian, setting); for previous work see $[25,29,27,48,41,5,42,43,44,31,14]$.

Remark. We can also extend our analysis to a class of slowly time-dependent external potentials without much additional work. We introduce a scale parameter, $\tau$, in time: $V(x, t):=W\left(\epsilon_{V} x, \tau t\right)$. To determine the size of $\tau$ heuristically we consider

$$
\frac{d}{d t} h(a, p, t)=p(\dot{p}+\nabla V(a, t))+\frac{1}{2}(\dot{a}-2 p) \cdot \nabla V(a, t)+\partial_{t} V(a, t) .
$$

We want the last two terms to have the same size. The second but last term is of size $\epsilon^{2} \epsilon_{V}$, since $\dot{a}$ satisfy the classical equations of motion to order $\epsilon^{2}$. The last term is of size $\tau$. Thus if $\tau$ is chosen to be $\tau=\mathcal{O}\left(\epsilon_{V}^{3}\right)$ all our estimates will survive.

The following example suggests that accelerating solitary wave solutions of Eq. (1.1) in a confining external potential can, in fact, survive for arbitrarily long times. Choose $V(x):=x \cdot A x+d \cdot x+c \geq 0$ and $A>0$ (positive matrix). Then (1.1) has the following solution:

$$
\psi(x, t)=\mathrm{e}^{\mathrm{i} p(t) \cdot(x-a(t))+\mathrm{i} \gamma(t))} \tilde{\eta}_{\mu}(x-a(t))
$$

with

$$
\dot{p}=-\nabla V(a), \quad \dot{a}=2 p, \quad \dot{\gamma}=p^{2}+\mu-V(a),
$$


where $\tilde{\eta}_{\mu}$ solves the equation

$$
-\Delta \eta+\mu \eta-f(\eta)+(x \cdot A x) \eta=0 .
$$

Thus, given a solution of the equations of motion (1.28), a family of solitary wave solutions is given by (1.27), for arbitrary times $t$. For details see Appendix D.

The first results of the above type, for bounded, time-independent potentials were proved in $[18,19]$ for the Hartree equation under a spectral assumption. This result was later extended to a general class of nonlinearities in [17]. Neither of these works deals with a confining external potential. In particular, their results do not extend to the longer time interval (1.25) described above. This type of results has also been obtained for the Korteweg-de Vries equation see [13, 14].

For local pure-power nonlinearities and a small parameter $\epsilon_{V}$, it has been shown in [4] that if an initial condition is of the form $\mathcal{S}_{a_{0} p_{0} \gamma_{0}} \eta_{\mu_{0}}$, then the solution $\psi(x, t)$ of Eq. (1.1) satisfies

$$
\epsilon_{V}^{-d}\left|\psi\left(\frac{x}{\epsilon_{V}}, \frac{t}{\epsilon_{V}}\right)\right|^{2} \rightarrow\left\|\eta_{\mu}\right\|_{L^{2}}^{2} \delta_{a(t)}
$$

in the $\mathrm{C}^{1 *}$ topology (dual to $\mathrm{C}^{1}$ ), provided $a(t)$ satisfies the equation $\frac{1}{2} \ddot{a}=\nabla W(a)$, where $V(x)=W\left(\epsilon_{V} x\right)$. This result was strengthened in [26] for a bounded external potential and in [8] for a potential given by a quadratic polynomial in $x$.

There have been many recent works on asymptotic properties for generalized nonlinear Schrödinger equations. Asymptotic stability, scattering and asymptotic completeness of solitary waves for bounded external potential tending to 0 at $\infty$ has been shown under rather stringent assumptions. See for example, $[37,38,39$, $5,6,10,11,7,45,46,47,12,35,40,23,33,20]$.

Though these are all-time results, where ours is long (but finite)-time, our approach has some advantages: we can handle confining potentials (for which the above-described results are meaningless); we require a much less stringent (and verifiable) spectral condition; we track the finite-dimensional soliton dynamics (Newton equations); and our methods are comparatively elementary.

Our paper is organized as follows. In Section 2, we state our hypotheses and the main result. In Section 3, we recall the Hamiltonian nature of Eq. (1.1) and describe symmetries of (1.1) for $V \equiv 0$. We give a precise definition of the soliton manifold $\mathrm{M}_{\mathrm{s}}$ and its tangent space. In Section 4 , we introduce a convenient parametrization of functions in a small neighborhood of $\mathrm{M}_{\mathrm{s}}$ in phase space, and we derive equations for the modulation parameters $\sigma=\{a, p, \gamma, \mu\}$ and the perturbation $w$ around a solitary wave $\eta_{\sigma}=\mathcal{S}_{a p \gamma} \eta_{\mu}$. In this parametrization, the perturbation $w$ is symplectically orthogonal to the tangent space $\mathrm{T}_{\eta_{\sigma}} \mathrm{M}_{\mathrm{s}}$ to $\mathrm{M}_{\mathrm{s}}$ at $\eta_{\sigma}$. In Section 5, we similarly decompose the initial condition $\psi_{0}$ deriving in this way the initial conditions, $\sigma_{0}$ and $w_{0}$, for $\sigma$ and $w$, and estimating $w_{0}$. In Section 6 , we derive bounds on the solitary wave position, $a$, and the momentum, $p$, by using the fact that the Hamiltonian, $h(a, p)$ is almost conserved in time. In Section 7, we construct the Lyapunov functional, $\Lambda(\psi, t)$, and compute its time derivative. This computation is used in Section 8 in order to obtain an upper bound on $\Lambda(\psi, t)$. 
This bound, together with the more difficult lower bound derived in Section 9, is used in Section 10 in order to estimate the perturbation $w$ and complete the proof of our main result, Theorem 2.1. Some basic inequalities are collected in Appendices A-C. In Appendix D, we construct a family of time-dependent solutions with parameters exactly satisfying the classical equations of motion.

\section{Notation, assumptions and main result}

Let $\mathrm{L}^{s}$ denote the usual Lebesgue space of functions, $\mathrm{C}^{s}$ the space of functions with $s$ continuous derivatives, and $\mathrm{H}_{s}$ the Sobolev space of order $s$. Abbreviate $\langle x\rangle^{2}:=1+|x|^{2}$.

Assumptions on the external potential. Let $W(x)$ be a $\mathrm{C}^{3}$ function, and let $\min _{x} W(x)=0$. Let $\beta \in \mathbb{Z}^{d}$ with $\beta_{j} \geq 0 \forall j=1, \ldots, d$ be a multi-index. Given a number $r \geq 1$ let $W$ be such that

$$
\begin{gathered}
\left|\partial_{x}^{\beta} W(x)\right| \leq C_{\max V}\langle x\rangle^{r-|\beta|}, \quad \text { for }|\beta| \leq 3, \\
\operatorname{Hess} W(x) \geq \rho_{1}\langle x\rangle^{r-2}
\end{gathered}
$$

and

$$
W(x) \geq c_{V}|x|^{r}, \quad \text { for }|x| \geq c_{L}
$$

for some positive constants $C_{\max V}, \rho_{1}, c_{V}, c_{L}$.

The number $r$ is called the growth rate of the external potential. Here Hess $W$ is the Hessian of $W$ with respect to spatial variables. Define $V(x):=W\left(\epsilon_{V} x\right)$. Then, for $r \geq 1$,

$$
\begin{gathered}
\left|\partial_{x}^{\beta} V(x)\right| \leq C_{V} \epsilon_{V}^{|\beta|}\left\langle\epsilon_{V} x\right\rangle^{r-|\beta|}, \quad \text { for }|\beta| \leq 3, \\
\operatorname{Hess} V(x) \geq \rho_{1} \epsilon_{V}^{2}\left\langle\epsilon_{V} x\right\rangle^{r-2}
\end{gathered}
$$

and

$$
V(x) \geq c_{V}\left(\epsilon_{V}|x|\right)^{r}, \quad \text { for } \epsilon_{V}|x| \geq c_{L} .
$$

Assumptions on the initial condition $\psi_{0}$. The energy space, $\mathscr{H}_{1, r}$, for a given growth rate $r$ of the external potential, is defined as

$$
\mathscr{H}_{1, r}:=\left\{\psi \in \mathrm{H}_{1}:\langle x\rangle^{r / 2} \psi \in \mathrm{L}^{2}\right\} .
$$

Let $\mathscr{H}_{1, r}^{\prime}$ denote the dual space of $\mathscr{H}_{1, r}$. The energy norm is defined as

$$
\|\psi\|_{\mathscr{H}_{1, r}}^{2}:=\|\psi\|_{\mathrm{H}_{1}}^{2}+\left\|\left\langle\epsilon_{V} x\right\rangle^{r / 2} \psi\right\|_{\mathrm{L}^{2}}^{2}
$$

since $|V| \leq C\left\langle\epsilon_{V} x\right\rangle^{r}$. We require $\psi_{0} \in \mathscr{H}_{1, r}$. It is convenient to include $\epsilon_{V}$ in the definition of the norm, since the spacial variable of the external potential scales with $\epsilon_{V}$. The naturalness of the norm (2.8) becomes manifest in, e.g., Propositions 8.1 and 9.1 .

In what follows, we identify complex functions with real two-component functions via

$$
\mathbb{C} \ni \psi(x)=\psi_{1}(x)+\mathrm{i} \psi_{2}(x) \longleftrightarrow \vec{\psi}(x)=\left(\psi_{1}(x), \psi_{2}(x)\right) \in \mathbb{R}^{2} .
$$


Consider a real function $F(\vec{\psi})$ on a space of real two-component functions, and let $F^{\prime}(\vec{\psi})$ denote its $L^{2}$-gradient. We identify this gradient with a complex function denoted by $F^{\prime}(\psi)$. Then

$$
F^{\prime}(\bar{\psi})=\overline{F^{\prime}(\psi)} \longleftrightarrow F(\sigma \vec{\psi})=F(\vec{\psi})
$$

where $\sigma:=\operatorname{diag}(1,-1)$, since the latter property is equivalent to $F^{\prime}(\vec{\psi})=\sigma F^{\prime}(\sigma \vec{\psi})$.

\section{Assumptions on the nonlinearity $f$.}

1. (GWP $[9,50,51,28])$ Equation (1.1) is globally well-posed in the space $\mathrm{C}\left(\mathbb{R}, \mathscr{H}_{1, r}\right) \cap \mathrm{C}^{1}\left(\mathbb{R}, \mathscr{H}_{1, r}^{\prime}\right)$. See Remark 2.1 below.

2. The nonlinearity $f$ maps from $\mathrm{H}_{1}$ to $\mathrm{H}_{-1}$, with $f(0)=0 . f(\psi)=F^{\prime}(\psi)$ is the $\mathrm{L}^{2}$-gradient of a $C^{3}$ functional $F: H_{1} \rightarrow \mathbb{R}$ defined on the space of real-valued, two-component functions, satisfying the following conditions:

(a) (Bounds)

$$
\sup _{\|u\|_{\mathrm{H}_{1}} \leq M}\left\|F^{\prime \prime}(u)\right\|_{\mathrm{B}\left(\mathrm{H}_{1}, \mathrm{H}_{-1}\right)}<\infty, \sup _{\|u\|_{\mathrm{H}_{1}} \leq M}\left\|F^{\prime \prime \prime}(u)\right\|_{\mathrm{H}_{1} \mapsto \mathrm{B}\left(\mathrm{H}_{1}, \mathrm{H}_{-1}\right)}<\infty,
$$

where $\mathrm{B}(X, Y)$ denotes the space of bounded linear operators from $X$ to $Y$.

(b) (Symmetries [17]) $F(\mathcal{T} \psi)=F(\psi)$ where $\mathcal{T}$ is either translation $\psi(x) \mapsto$ $\psi(x+a) \forall a \in \mathbb{R}^{d}$, or spatial rotation $\psi(x) \mapsto \psi\left(R^{-1} x\right), \forall R \in \mathrm{SO}(d)$, or boosts $\mathcal{T}_{p}^{\mathrm{b}}: u(x) \mapsto \mathrm{e}^{\mathrm{i} p \cdot x} u(x), \forall p \in \mathbb{R}^{d}$, or gauge transformations $\psi \mapsto \mathrm{e}^{\mathrm{i} \gamma} \psi, \forall \gamma \in \mathbb{S}^{1}$, or complex conjugation $\psi \mapsto \bar{\psi}$.

3. (Solitary waves) There exists a bounded open interval $\tilde{I}$ on the positive real axis such that for all $\mu \in \tilde{I}$ :

(a) (Ground state $[3,1,2,30])$ The equation

$$
-\Delta \psi+\mu \psi-f(\psi)=0
$$

has a spherically symmetric, positive $\mathrm{L}^{2} \cap \mathrm{C}^{2}$ solution, $\eta=\eta_{\mu}$.

(b) (Stability: see, e.g., $[49,21]$ ) This solution, $\eta$, satisfies

$$
\partial_{\mu} \int \eta_{\mu}^{2} \mathrm{~d}^{d} x>0
$$

(c) (Null space condition: see, e.g., [17] and also [48]) Let $\mathcal{L}_{\eta}$ be the linear operator

$$
\mathcal{L}_{\eta}:=\left(\begin{array}{cc}
L_{1} & 0 \\
0 & L_{2}
\end{array}\right)
$$

where $L_{1}:=-\Delta+\mu-f^{(1)}(\eta)$, and $L_{2}:=-\Delta+\mu-f^{(2)}(\eta)$, with $f^{(1)}:=\left(\partial_{\operatorname{Re} \psi}(\operatorname{Re}(f))\right)(\eta)$, and $f^{(2)}:=\left(\partial_{\operatorname{Im} \psi}(\operatorname{Im}(f))\right)(\eta)$. We require that

$$
\mathrm{N}\left(\mathcal{L}_{\eta}\right)=\operatorname{span}\left\{\left(\begin{array}{l}
0 \\
\eta
\end{array}\right),\left(\begin{array}{c}
\partial_{x_{j}} \eta \\
0
\end{array}\right), j=1, \ldots, d\right\}
$$


Conditions $2-3$ on the nonlinearity are discussed in [17], where further references can be found. Examples of nonlinearities that satisfy the above requirements are local nonlinearities

$$
f(\psi)=\beta|\psi|^{s_{1}} \psi+\lambda|\psi|^{s_{2}} \psi, \quad 0<s_{1}<s_{2}<\frac{4}{d}, \beta \in \mathbb{R}, \lambda>0,
$$

and Hartree nonlinearities

$$
f(\psi)=\lambda\left(\Phi *|\psi|^{2}\right) \psi, \quad \lambda>0,
$$

where $\Phi$ is of positive type, continuous and spherically symmetric and tends to 0 , as $|x| \rightarrow \infty$. Of course, $\lambda$ can be scaled out by rescaling $\psi$. For precise conditions on $\Phi$ we refer to $[9,28]$.

Remark 2.1. For Hartree nonlinearities global well-posedness is known for potentials $0 \leq V \in \mathrm{L}_{\text {loc }}^{1}$ [28]. For local nonlinearities, the situation is more delicate. Global well-posedness and energy conservation is known for potentials with growthrate $r \leq 2$ [9]. For $r>2$ and local nonlinearities, local well-posedness has been shown in the energy space $[50,51]$. For local nonlinearities, a proof of the energy conservation needed for global well-posedness, and the application of this theory to our results, is missing.

For $V \equiv 0$, Eq. (1.1) is the usual generalized nonlinear Schrödinger (or Hartree) equation. For self-focusing nonlinearities as in examples (2.14) and (2.15), it has stable solitary wave solutions of the form

$$
\eta_{\sigma(t)}(x):=\mathrm{e}^{\mathrm{i} p(t) \cdot(x-a(t))+\mathrm{i} \gamma(t)} \eta_{\mu(t)}(x-a(t)),
$$

where $\sigma(t):=\{a(t), p(t), \gamma(t), \mu(t)\}$, and

$$
a(t)=2 p t+a, \gamma(t)=\mu t+p^{2} t+\gamma, p(t)=p, \quad \mu(t)=\mu,
$$

with $\gamma \in \mathbb{S}^{1}, a, p \in \mathbb{R}^{d}$ and $\mu \in \mathbb{R}^{+}$, and where $\eta_{\mu}$ is the spherically symmetric, positive solution of the nonlinear eigenvalue problem

$$
-\Delta \eta+\mu \eta-f(\eta)=0 .
$$

Recall from (1.6) that the linear map $\mathcal{S}_{a p \gamma}$ is defined as

$$
\left(\mathcal{S}_{a p \gamma} g\right)(x):=\mathrm{e}^{\mathrm{i} p \cdot(x-a)+\mathrm{i} \gamma} g(x-a) .
$$

In analyzing solitary wave solutions to (1.1) we encounter two length scales: the size $\propto \mu^{-1 / 2}$ of the support of the function $\eta_{\mu}$, which is determined by our choice of initial condition $\psi_{0}$, and a length scale determined by the potential, $V$, measured by the small parameter $\epsilon_{V}$. We consider the regime,

$$
\frac{\epsilon_{V}}{\sqrt{\mu}} \ll 1
$$

We claim in the introduction that if $\psi_{0}$ is close to $\eta_{\sigma}$, for some $\sigma$ then we retain control for times $\propto \epsilon^{-1}$. Restricting the initial condition to a smaller class of $\eta_{\sigma}$, with small initial energy, we retain control for longer times. In our main 
theorem, which proves this claim, we wish to treat both cases uniformly. To this end, let $\epsilon_{h}$ and $K$ be positive numbers such that $\epsilon_{h} \in K\left[\epsilon_{V}, \min _{\mu \in I} \sqrt{\mu}\right]$ and assume

$$
h\left(a_{0}, p_{0}\right):=\frac{1}{2}\left(p_{0}^{2}+V(a)\right) \leq \epsilon_{h}
$$

(recall $\min _{a} V(a)=0$ ). The lower bound for $\epsilon_{h}$ corresponds to our restricted class of initial data, the upper bound to the larger class of data. In particular, $\epsilon_{h} \geq K \epsilon_{V}$. $I \subset \tilde{I}$.

We are now ready to state our main result. Fix an open proper sub-interval

Theorem 2.1. Let $f$ and $V$ satisfy the conditions listed above. There exists $T>0$ such that for $\epsilon:=\epsilon_{V}+\epsilon_{0}$ sufficiently small, and $\epsilon_{h} \geq K \epsilon_{V}$, if the initial condition $\psi_{0}$ satisfies

$$
\left\|\psi_{0}-\mathcal{S}_{a_{0} p_{0} \gamma_{0}} \eta_{\mu_{0}}\right\|_{\mathrm{H}_{1}}+\left\|\left\langle\epsilon_{V} x\right\rangle^{r / 2}\left(\psi_{0}-\mathcal{S}_{a_{0} p_{0} \gamma_{0}} \eta_{\mu_{0}}\right)\right\|_{\mathrm{L}^{2}} \leq \epsilon_{0}
$$

for some $\sigma_{0}:=\left\{a_{0}, p_{0}, \gamma_{0}, \mu_{0}\right\} \in \mathbb{R}^{d} \times \mathbb{R}^{d} \times \mathbb{S}^{1} \times I$ such that

$$
h\left(a_{0}, p_{0}\right) \leq \epsilon_{h},
$$

then for times $0 \leq t \leq T\left(\epsilon_{V} \sqrt{\epsilon_{h}}+\epsilon^{2}\right)^{-1}$, the solution to Eq. (1.1) with this initial condition is of the form

$$
\psi(x, t)=\mathcal{S}_{a(t) p(t) \gamma(t)}\left(\eta_{\mu(t)}(x)+w(x, t)\right),
$$

where $\|w\|_{\mathrm{H}_{1}}+\left\|\left\langle\epsilon_{V} x\right\rangle^{r / 2} w\right\|_{\mathrm{L}^{2}} \leq C \epsilon$. The modulation parameters $a, p, \gamma$ and $\mu$ satisfy the differential equations

$$
\begin{aligned}
& \dot{p}=-(\nabla V)(a)+\mathcal{O}\left(\epsilon^{2}\right), \\
& \dot{a}=2 p+\mathcal{O}\left(\epsilon^{2}\right), \\
& \dot{\gamma}=\mu-V(a)+p^{2}+\mathcal{O}\left(\epsilon^{2}\right), \\
& \dot{\mu}=\mathcal{O}\left(\epsilon^{2}\right) .
\end{aligned}
$$

Remark 2.2 (Remark about notation). Fréchet derivatives are always understood to be defined on real spaces. They are denoted by primes. $C$ and $c$ denote various constants that often change between consecutive lines and which do not depend on $\epsilon_{V}, \epsilon_{0}$ or $\epsilon$.

\section{Soliton manifold}

In this section we recall the Hamiltonian nature of Eq. (1.1) and some of its symmetries. We also define the soliton manifold and its tangent space.

An important part in our approach is played by the variational character of (1.1). More precisely, the nonlinear Schrödinger equation (1.1) is a Hamiltonian system with Hamiltonian

$$
\mathcal{H}_{V}(\psi):=\frac{1}{2} \int\left(|\nabla \psi|^{2}+V|\psi|^{2}\right) \mathrm{d}^{d} x-F(\psi) .
$$


The Hamiltonian $\mathcal{H}_{V}$ is conserved, i.e.,

$$
\mathcal{H}_{V}(\psi)=\mathcal{H}_{V}\left(\psi_{0}\right)
$$

A proof of this can be found, for local nonlinearities and $r \leq 2$, in, e.g., Cazenave [9], and for Hartree nonlinearities in [28]. An important role is played by the mass

$$
\mathcal{N}(\psi):=\int|\psi|^{2} \mathrm{~d}^{d} x
$$

which also is conserved,

$$
\mathcal{N}(\psi(t))=\mathcal{N}\left(\psi_{0}\right) .
$$

We often identify complex spaces, such as the Sobolev space $\mathrm{H}_{1}\left(\mathbb{R}^{d}, \mathbb{C}\right)$, with real spaces; e.g., $\mathrm{H}_{1}\left(\mathbb{R}^{d}, \mathbb{R}^{2}\right)$, using the identification $\psi=\psi_{1}+\mathrm{i} \psi_{2} \leftrightarrow\left(\psi_{1}, \psi_{2}\right)=: \vec{\psi}$. With this identification, the complex structure $\mathrm{i}^{-1}$ corresponds to the operator

$$
J:=\left(\begin{array}{cc}
0 & 1 \\
-1 & 0
\end{array}\right)
$$

The real $\mathrm{L}^{2}$-inner product in the real notation is

$$
\langle\vec{u}, \vec{w}\rangle:=\int\left(u_{1} w_{1}+u_{2} w_{2}\right) \mathrm{d}^{d} x,
$$

where $\vec{u}:=\left(u_{1}, u_{2}\right)$. In the complex notation it becomes

$$
\langle u, w\rangle:=\operatorname{Re} \int u \bar{w} \mathrm{~d}^{d} x .
$$

We henceforth abuse notation and drop the arrows. The symplectic form is

$$
\omega(u, w):=\operatorname{Im} \int u \bar{w} \mathrm{~d}^{d} x .
$$

We note that $\omega(u, w)=\left\langle u, J^{-1} v\right\rangle$ in the real notation.

Equation (1.1) with $V \equiv 0$ is invariant under spatial translations, $\mathcal{T}_{a}^{\text {tr }}$, gauge transformations, $\mathcal{T}_{\gamma}^{\mathrm{g}}$, and boost transformations, $\mathcal{T}_{p}^{\text {boost }}$, where

$$
\begin{gathered}
\mathcal{T}_{a}^{\mathrm{tr}}: \psi(x, t) \mapsto \psi(x-a, t), \quad \mathcal{T}_{\gamma}^{\mathrm{g}}: \psi(x, t) \mapsto \mathrm{e}^{\mathrm{i} \gamma} \psi(x, t), \\
\mathcal{T}_{p}^{\text {boost }}: \psi(x, t) \mapsto \mathrm{e}^{\mathrm{i}\left(p \cdot x-p^{2} t\right)} \psi(x-2 p t, t)
\end{gathered}
$$

The transformations (3.9)-(3.10) map solutions of Eq. (1.1) with $V \equiv 0$ into solutions of (1.1) with $V \equiv 0$.

Let $\mathcal{T}_{p}^{\mathrm{b}}: \psi(x) \mapsto \mathrm{e}^{\mathrm{i} p \cdot x} \psi(x)$ be the $t=0$ slice of the boost transform. The combined symmetry transformations $\mathcal{S}_{a p \gamma}$ introduced in (2.19) can be expressed as

$$
\mathcal{S}_{a p \gamma} \eta=\mathcal{T}_{a}^{\mathrm{tr}} \mathcal{T}_{p}^{\mathrm{b}} \mathcal{T}_{\gamma}^{\mathrm{g}} \eta_{\mu}(x)=\mathrm{e}^{\mathrm{i}(p \cdot(x-a)+\gamma)} \eta_{\mu}(x-a) .
$$

We define the soliton manifold as

$$
\mathrm{M}_{\mathrm{s}}:=\left\{\mathcal{S}_{a p \gamma} \eta_{\mu}:\{a, p, \gamma, \mu\} \in \mathbb{R}^{d} \times \mathbb{R}^{d} \times \mathbb{S}^{1} \times I\right\} .
$$


The tangent space to this manifold at the solitary wave profile $\eta_{\mu} \in \mathrm{M}_{\mathrm{s}}$ is given by

$$
\mathrm{T}_{\eta_{\mu}} \mathrm{M}_{\mathrm{s}}=\operatorname{span}\left(z_{\mathrm{t}}, z_{\mathrm{g}}, z_{\mathrm{b}}, z_{\mathrm{s}}\right)
$$

where

$$
\begin{array}{ll}
z_{\mathrm{t}}:=\left.\nabla_{a} \mathcal{T}_{a}^{\mathrm{tr}} \eta_{\mu}\right|_{a=0}=\left(\begin{array}{c}
-\nabla \eta_{\mu} \\
0
\end{array}\right), & z_{\mathrm{g}}:=\left.\frac{\partial}{\partial \gamma} \mathcal{T}_{\gamma}^{\mathrm{g}} \eta_{\mu}\right|_{\gamma=0}=\left(\begin{array}{c}
0 \\
\eta_{\mu}
\end{array}\right), \\
z_{\mathrm{b}}:=\left.\nabla_{p} \mathcal{T}_{p}^{\text {boost }} \eta_{\mu}\right|_{p=0, t=0}=\left(\begin{array}{c}
0 \\
x \eta_{\mu}
\end{array}\right), & z_{\mathrm{s}}:=\left(\begin{array}{c}
\partial_{\mu} \eta_{\mu} \\
0
\end{array}\right) .
\end{array}
$$

Above, we have explicitly written the basis of tangent vectors in the real space.

Recall that the equation $(2.10)$ can be written as $\mathcal{E}_{\mu}^{\prime}\left(\eta_{\mu}\right)=0$ where

$$
\mathcal{E}_{\mu}(\psi)=\mathcal{H}_{V \equiv 0}(\psi)+\frac{\mu}{2} \mathcal{N}(\psi)
$$

Then the tangent vectors listed above are generalized zero modes of the operator $J \mathcal{L}_{\mu}$, where $\mathcal{L}_{\mu}:=\mathcal{E}_{\mu}^{\prime \prime}\left(\eta_{\mu}\right)$. That is, $\left(J \mathcal{L}_{\mu}\right)^{2} z=0$ for each tangent vector $z$ above. To see this fact for $z_{\mathrm{g}}$, for example, recall that $\mathcal{E}_{\mu}^{\prime}(\psi)$ is gauge-invariant. Hence $\mathcal{E}_{\mu}^{\prime}\left(\mathcal{T}_{\gamma}^{\mathrm{g}} \eta_{\mu}\right)=0$. Taking the derivative with respect to the parameter $\gamma$ at $\gamma=0$ gives $\mathcal{L}_{\eta} z_{\mathrm{g}}=0$. The other relations are derived analogously (see [48]).

\section{Symplectically orthogonal decomposition}

In this section we make a change of coordinates for the Hamiltonian system $\psi \mapsto(\sigma, w)$, where $\sigma:=(a, p, \gamma, \mu)$. We also give the equations in this new set of coordinates.

Let

$$
m(\mu):=\frac{1}{2} \int \eta_{\mu}^{2}(x) \mathrm{d}^{d} x
$$

Let

$$
C_{I}:=\max _{z \in\left\{x \eta_{\mu}, \eta_{\mu}, \nabla \eta_{\mu}, \partial_{\mu} \eta_{\mu}\right\}}\left(\|z\|_{\mathrm{H}_{1}},\left\|\left\langle\epsilon_{V} x\right\rangle^{r / 2} z\right\|_{\mathrm{L}^{2}},\|\mathcal{K} z\|_{\mathrm{L}^{2}}\right) .
$$

When it will not cause confusion, for $\sigma=\{a, p, \gamma, \mu\}$ we will abbreviate

$$
\eta_{\sigma}:=\mathcal{S}_{a p \gamma} \eta_{\mu}
$$

Now define the neighborhood of $\mathrm{M}_{\mathrm{s}}$ :

$$
U_{\delta}:=\left\{\psi \in L^{2}: \inf _{\sigma \in \Sigma}\left\|\psi-\eta_{\sigma}\right\|_{\mathrm{L}^{2}} \leq \delta\right\}
$$

where $\Sigma:=\left\{a, p, \gamma, \mu: a \in \mathbb{R}^{d}, p \in \mathbb{R}^{d}, \gamma \in \mathbb{S}^{1}, \mu \in I\right\}$. Our goal is to decompose a given function $\psi \in U_{\delta}$ into a solitary wave and a perturbation:

$$
\psi=\mathcal{S}_{a p \gamma}\left(\eta_{\mu}+w\right) \text {. }
$$

We do this according to the following theorem. Let $\tilde{\Sigma}:=\left\{a, p, \gamma, \mu: a \in \mathbb{R}^{d}, p \in\right.$ $\left.\mathbb{R}^{d}, \gamma \in \mathbb{S}^{1}, \mu \in \tilde{I}\right\}$. 
Theorem 4.1. There exists $\delta>0$ and a unique map $\varsigma \in \mathrm{C}^{1}\left(U_{\delta}, \tilde{\Sigma}\right)$ such that (i)

$$
\left\langle\psi-\eta_{\varsigma(\psi)}, J^{-1} z\right\rangle=0, \quad \forall z \in \mathrm{T}_{\eta_{\varsigma(\psi)}} \mathrm{M}_{\mathrm{s}}, \quad \forall \psi \in U_{\delta}
$$

and (ii) if, in addition, $\delta \ll\left(2 C_{I}\right)^{-1} \min \left(m(\mu), m^{\prime}(\mu)\right)$ then there exists a constant $c_{I}$ independent of $\delta$ such that

$$
\sup _{\psi \in U_{\delta}}\left\|\varsigma^{\prime}(\psi)\right\|_{L^{2}} \leq c_{I}
$$

Proof. Part (i): Let the map $G: L^{2} \times \tilde{\Sigma} \mapsto \mathbb{R}^{2 d+2}$ be defined by

$$
G_{j}(\psi, \varsigma):=\left\langle\psi-\eta_{\varsigma}, J^{-1} z_{\varsigma, j}\right\rangle, \quad \forall j=1, \ldots 2 d+2 .
$$

Part (i) is proved by applying the implicit function theorem to the equation $G(\psi, \varsigma)=0$, around a point $\left(\eta_{\sigma}, \sigma\right)$. For details we refer to Proposition 5.1 in [17].

Part (ii): Abbreviate:

$$
\Omega_{j k}:=\left\langle\partial_{\varsigma_{j}} \eta_{\varsigma}, J^{-1} z_{\varsigma, k}\right\rangle
$$

where $z_{\varsigma, k}$ is the $k$ :th element of $\mathcal{S}_{a p \gamma}\left\{z_{\mathrm{t}}, z_{\mathrm{g}}, z_{\mathrm{b}}, z_{\mathrm{s}}\right\}$. By explicitly inserting the tangent vectors, we find that $\|\Omega\|_{\mathrm{L}^{2}} \geq \inf _{\mu \in I}\left(m(\mu), m^{\prime}(\mu)\right)$. Thus, $\Omega$ is invertible by Condition 3b in Section 2.

From a variation of $\psi$ in $G(\psi, \varsigma(\psi))=0$ we find

$$
\varsigma_{k}^{\prime}(\psi)=\sum_{j=1}^{2 d+2}\left(J^{-1} z_{\varsigma}\right)_{j}\left(\tilde{\Omega}^{-1}\right)_{j k},
$$

where

$$
\tilde{\Omega}_{j k}:=\Omega_{j k}+\left\langle\psi-\eta_{\varsigma(\psi)}, J^{-1} \partial_{\varsigma_{j}} z_{\varsigma, k}\right\rangle
$$

Using the upper bound of $\delta$, and the definition of $C_{I}$ above, we find

$$
\sup _{\psi \in U_{\delta}}\left\|\varsigma^{\prime}(\psi)\right\|_{\mathrm{L}^{2}} \leq \frac{2 C_{I}}{\inf _{\mu \in I}\left(m(\mu), m^{\prime}(\mu)\right)}=: c_{I} .
$$

We now assume $\psi(t) \in U_{\delta} \cap \mathscr{H}_{1, r}$, and set $\sigma(t):=\varsigma(\psi(t))$ as defined by Theorem 4.1. Write

so that $w$ satisfies

$$
u:=\mathcal{S}_{a p \gamma}^{-1} \psi=\eta_{\mu}+w
$$

$$
\left\langle w, J^{-1} z\right\rangle=0, \quad \forall z \in \mathrm{T}_{\eta_{\mu}} \mathrm{M}_{\mathrm{s}} .
$$

Here $u$ is the solution in a moving frame.

Denote the anti-self-adjoint infinitesimal generators of symmetries as

$$
\mathcal{K}_{j}=\partial_{x_{j}}, \mathcal{K}_{d+j}=\mathrm{i} x_{j}, \mathcal{K}_{2 d+1}=\mathrm{i}, \mathcal{K}_{2 d+2}=\partial_{\mu}, \quad j=1, \ldots, d
$$

and define corresponding coefficients

$$
\begin{gathered}
\alpha_{j}=\dot{a}_{j}-2 p_{j}, \quad \alpha_{d+j}=-\dot{p}_{j}-\partial_{x_{j}} V(a), \quad j=1, \ldots, d, \\
\alpha_{2 d+1}=\mu-p^{2}+\dot{a} \cdot p-V(a)-\dot{\gamma}, \quad \alpha_{2 d+2}=-\dot{\mu} .
\end{gathered}
$$


Denote

$$
\underline{\alpha} \cdot \underline{\mathcal{K}}:=\sum_{j=1}^{2 d+1} \alpha_{j} \mathcal{K}_{j}, \quad \text { and } \quad \alpha \cdot \mathcal{K}:=\underline{\alpha} \cdot \underline{\mathcal{K}}+\alpha_{2 d+2} \partial_{\mu} .
$$

Substituting $\psi=\mathcal{S}_{a p \gamma} u$ into (1.1) we obtain

$$
\mathrm{i} \dot{u}=\mathcal{E}_{\mu}^{\prime}(u)+\mathcal{R}_{V} u+\underline{\mathrm{i}} \underline{\alpha} \cdot \underline{\mathcal{K}} u,
$$

where

$$
\mathcal{R}_{V}(x)=V(x+a)-V(a)-\nabla V(a) \cdot x .
$$

To obtain the equations for $(\sigma, w)$ we project Eqn. (4.18) onto $\mathrm{T}_{\eta} \mathrm{M}_{\mathrm{s}}$ and $\left(J \mathrm{~T}_{\eta} \mathrm{M}_{\mathrm{s}}\right)^{\perp}$ and use (4.12). We illustrate this method of deriving the equations for $\sigma$, for the projection of (4.18) along i $\eta$ :

$$
\left\langle\eta, \dot{\mu} \partial_{\mu} \eta+\dot{w}\right\rangle=\left\langle\mathrm{i} \eta, \mathcal{L}_{\eta} w+N_{\eta}(w)+\mathcal{R}_{V}(\eta+w)+\mathrm{i} \underline{\alpha} \cdot \underline{\mathcal{K}}(\eta+w)\right\rangle .
$$

where we have used $u=\eta+w$ and $\mathcal{E}_{\mu}^{\prime}(u)=\mathcal{L}_{\eta} w+N_{\eta}(w)$ where $\mathcal{L}_{\eta}:=\mathcal{E}_{\mu}^{\prime \prime}(\eta)$ is given explicitly as

$$
\mathcal{L}_{\eta} w=-\Delta w+\mu w-f^{\prime}(\eta) w .
$$

In particular, for local nonlinearities of the form $g\left(|\psi|^{2}\right) \psi$, we have in the complex notation, since $\eta(x) \in \mathbb{R}$,

$$
\mathcal{L}_{\eta} w:=-\Delta w+\mu w-g\left(\eta^{2}\right) w-2 \eta g^{\prime}\left(\eta^{2}\right) \operatorname{Re} w .
$$

Here

$$
N_{\eta}(w):=-f(\eta+w)+f(\eta)+f^{\prime}(\eta) w .
$$

We find the equation for $\dot{\mu}$ once we note that $\partial_{t}\langle\eta, w\rangle=0, \mathcal{L}_{\eta} \mathrm{i} \eta=0,\left\langle\mathrm{i} \eta, \mathcal{R}_{V} \eta\right\rangle=0$, $\langle\eta, \underline{\mathcal{K}} \eta\rangle=0$ and $\underline{\mathcal{K}}^{*}=-\underline{\mathcal{K}}$. Inserting this into (4.20) gives

$$
\dot{\mu} m^{\prime}(\mu)=\left\langle i \eta, N_{\eta}(w)+\mathcal{R}_{V} w\right\rangle-\alpha \cdot\langle\mathcal{K} \eta, w\rangle .
$$

The projection along the other directions works the same way: we use the fact that these directions are the generalized zero modes of $\mathcal{L}_{\eta}$, and furthermore that they are orthogonal to $J w$. The calculations are worked out in detail in [17] (see Eqns. (6.20)-(6.22) in [17]). We give the result:

$$
\begin{gathered}
\dot{\gamma}=\mu-p^{2}+\dot{a} \cdot p-V(a)-\left(m^{\prime}(\mu)\right)^{-1}\left(\left\langle\partial_{\mu} \eta, N_{\eta}(w)+\mathcal{R}_{V} w\right\rangle\right. \\
\left.-\alpha \cdot\left\langle\mathcal{K} \partial_{\mu} \eta, \mathrm{i} w\right\rangle+\left\langle\partial_{\mu} \eta, \mathcal{R}_{V} \eta\right\rangle\right), \\
\dot{\mu}=\left(m^{\prime}(\mu)\right)^{-1}\left(\left\langle\mathrm{i} \eta, N_{\eta}(w)+\mathcal{R}_{V} w\right\rangle-\alpha \cdot\langle\mathcal{K} \eta, w\rangle\right), \\
\dot{a}_{k}=2 p_{k}+\left(m(\mu)^{-1}\right)\left(\left\langle\mathrm{i} x_{k} \eta, N_{\eta}(w)+\mathcal{R}_{V} w\right\rangle-\alpha \cdot\left\langle\mathcal{K} x_{k} \eta, w\right\rangle\right), \\
\dot{p}_{k}=-\partial_{a_{k}} V(a)+(m(\mu))^{-1}\left(-\frac{1}{2}\left\langle\left(\partial_{x_{k}} \mathcal{R}_{V}\right) \eta, \eta\right\rangle+\left\langle\partial_{k} \eta, N_{\eta}(w)+\mathcal{R}_{V} w\right\rangle\right. \\
\left.-\alpha \cdot\left\langle\mathcal{K} \partial_{k} \eta, \mathrm{i} w\right\rangle\right),
\end{gathered}
$$

and

$$
\mathrm{i} \dot{w}=\mathcal{L}_{\eta} w+N(w)+\mathcal{R}_{V}(\eta+w)+\mathrm{i} \underline{\alpha} \cdot \underline{\mathcal{K}}(\eta+w)-\mathrm{i} \dot{\mu} \partial_{\mu} \eta .
$$


Note that the first two terms on the right-hand side of Eqn. (4.28) can be written as $-\partial_{a_{k}} V_{\text {eff }}(a, \mu)$, where

$$
V_{\text {eff }}(a, \mu):=\left\|\eta_{\mu}\right\|_{\mathrm{L}^{2}}^{-2} \int V(a+x)\left|\eta_{\mu}(x)\right|^{2} \mathrm{~d}^{d} x .
$$

Hence,

$$
\dot{p}_{k}=-\nabla_{a} V_{\mathrm{eff}}(a, \mu)+\left(m(\mu)^{-1}\left\langle\partial_{x_{k}} \eta_{\mu}, N_{\eta}(w)\right\rangle+\mathcal{O}\left(\|w\|_{L^{2}}\left(\epsilon_{V}^{2}+|\alpha|\right)\right),\right.
$$

where $|\alpha|^{2}=\sum\left|\alpha_{j}\right|^{2}$.

Thus we have obtained the dynamical equations for $(\sigma, w)$.

Remark 4.1. Related equations were derived in [48] for the perturbation of the classical NLS equation by a more general nonlinearity (see also [41] for a study of the nonlinear wave equation).

Remark 4.2. The transformation

$$
\sigma:=(a, p, \gamma, \mu) \mapsto \hat{\sigma}:=(a, P, \gamma, m)
$$

with $P:=\frac{1}{2} p\left\|\eta_{\mu}\right\|_{\mathrm{L}^{2}}^{2}$ and $m:=\frac{1}{2}\left\|\eta_{\mu}\right\|_{\mathrm{L}^{2}}^{2}$ gives a canonical symplectic structure and Darboux coordinates on $\mathrm{M}_{\mathrm{s}}$, i.e., for $w=0$

$$
\begin{array}{ll}
\dot{P}=-\partial_{a} \mathcal{H}_{V}\left(\mathcal{S}_{a p \gamma} \eta_{\mu}\right), & \dot{a}=\partial_{P} \mathcal{H}_{V}\left(\mathcal{S}_{a p \gamma} \eta_{\mu}\right), \\
\dot{m}=\partial_{\gamma} \mathcal{H}_{V}\left(\mathcal{S}_{a p \gamma} \eta_{\mu}\right), & \dot{\gamma}=-\partial_{m} \mathcal{H}_{V}\left(\mathcal{S}_{a p \gamma} \eta_{\mu}\right) .
\end{array}
$$

Here $\nabla_{\hat{\sigma}} \mathcal{H}_{V}\left(\mathcal{S}_{a p \gamma} \eta_{\mu}\right)=\left(m \nabla_{a} V_{\text {eff }}, 2 P / m, 0,-P^{2} / m^{2}+V(a)-\mu\right)$.

\section{Initial conditions $\tilde{\sigma}_{0}, w_{0}$.}

In this section we use Theorem 4.1 in order to decompose the initial condition $\psi_{0}$ as (see Figure 5.1)

$$
\psi_{0}=\mathcal{S}_{\tilde{a}_{0}, \tilde{p}_{0}, \tilde{\gamma}_{0}}\left(\eta_{\tilde{\mu}_{0}}+w_{0}\right)
$$

so that $w_{0} \perp J^{-1} \mathrm{~T}_{\eta_{\tilde{\mu}_{0}}} \mathrm{M}_{\mathrm{s}}$. This decomposition provides the initial conditions $\tilde{\sigma}_{0}$ and $w_{0}$, for the parameters, $\sigma$, and fluctuation, $w$ (determined for later times by Theorem 4.1). The main work here goes into estimating $w_{0}$. Let $\varsigma: U_{\delta} \mapsto \tilde{\Sigma}$ be the map established in Theorem 4.1. Then $\tilde{\sigma}_{0}=\left\{\tilde{a}_{0}, \tilde{p}_{0}, \tilde{\gamma}_{0}, \tilde{\mu}_{0}\right\}$ and $w_{0}$ are given as $\tilde{\sigma}_{0}:=\varsigma\left(\psi_{0}\right)$ and

$$
w_{0}:=\mathcal{S}_{\tilde{a}_{0} \tilde{p}_{0} \tilde{\gamma}_{0}}^{-1}\left(\psi_{0}-\eta_{\tilde{\sigma}_{0}}\right), \quad w_{0} \perp J \mathrm{~T}_{\eta_{\tilde{\mu}_{0}}} \mathrm{M}_{\mathrm{s}} .
$$

Recall the definitions of $\mathcal{K}(4.14)$, and $C_{I}$ (4.2). From Theorem 4.1 we have $\sup _{\psi \in U_{\delta}}\left\|\varsigma^{\prime}(\psi)\right\|_{\mathrm{L}^{2}} \leq c_{I}$.

Bounds for $w_{0}$ and $\tilde{\sigma}_{0}$ are stated in the following proposition. 


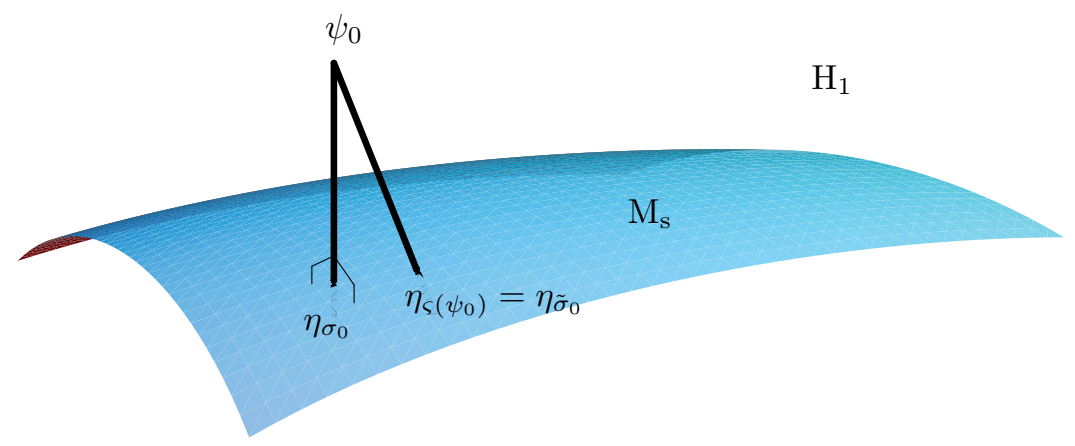

FiguRE 5.1. Orthogonal decomposition versus skew-orthogonal decomposition.

Proposition 5.1. Let $w_{0}$ be defined as above. Let $\sigma_{0}:=\left\{a_{0}, p_{0}, \gamma_{0}, \mu_{0}\right\}$ and let $\psi_{0}$ satisfy $\left\|\psi_{0}-\eta_{\sigma_{0}}\right\|_{L^{2}} \leq \delta$ (where $\delta$ is from Theorem 4.1), and let $\psi_{0} \in \mathscr{H}_{1, r}$. Then there exists positive constants $C_{1}, C_{2}$, such that

$$
\begin{aligned}
\left|\tilde{\sigma}_{0}-\sigma_{0}\right| & \leq c_{I}\left\|\psi_{0}-\eta_{\sigma_{0}}\right\|_{\mathrm{L}^{2}}, \\
\left\|w_{0}\right\|_{\mathrm{H}_{1}} & \leq C_{1}\left(1+p_{0}^{4}+\left\|\psi_{0}-\eta_{\sigma_{0}}\right\|_{\mathrm{L}^{2}}^{4}\right)\left\|\psi_{0}-\eta_{\sigma_{0}}\right\|_{\mathrm{H}_{1}}
\end{aligned}
$$

and

$$
\begin{aligned}
& \left\|\left\langle\epsilon_{V} x\right\rangle^{r / 2} w_{0}\right\|_{\mathrm{L}^{2}} \leq 3^{r / 2}\left\|\left\langle\epsilon_{V} x\right\rangle^{r / 2}\left(\psi_{0}-\eta_{\sigma_{0}}\right)\right\|_{\mathrm{L}^{2}} \\
& +C_{2}\left(1+\left|p_{0}\right|^{2}+\epsilon_{V}^{r}\left|a_{0}\right|^{r}+\left\|\psi_{0}-\eta_{\sigma_{0}}\right\|_{\mathrm{L}^{2}}^{2}+\epsilon_{V}^{r}\left\|\psi_{0}-\eta_{\sigma_{0}}\right\|_{\mathrm{L}^{2}}^{r}\right)\left\|\psi_{0}-\eta_{\sigma_{0}}\right\|_{\mathrm{L}^{2}},
\end{aligned}
$$

where $C_{1}$ and $C_{2}$ depend only on $C_{I}, c_{I}$ and $r$, where $C_{I}$ is defined in (4.2) and $c_{I}$ in Theorem 4.1 .

Proof. First we consider inequality (5.3). Abbreviate $\tilde{\sigma}_{0}:=\varsigma\left(\psi_{0}\right)$ and analogously for the components $a, p, \gamma, \mu$ of $\varsigma$. Let $|\varsigma|^{2}:=\sum_{j=1}^{2 d+2}\left|\varsigma_{j}\right|^{2}$. From Theorem 4.1 we know that $\varsigma(\psi)$ is a $\mathrm{C}^{1}$-map. Thus, for $j \in 1, \ldots, 2 d+2$ and some $\theta_{1} \in[0,1]$

$$
\left(\tilde{\sigma}_{0}-\sigma_{0}\right)_{j}=\left\langle\varsigma_{j}^{\prime}\left(\theta_{1} \psi_{0}+\left(1-\theta_{1}\right) \eta_{\sigma_{0}}\right),\left(\psi_{0}-\eta_{\sigma_{0}}\right)\right\rangle .
$$

Since $\sup _{\psi \in U_{\delta}}\left\|\varsigma^{\prime}(\psi)\right\| \leq c_{I}$ the inequality (5.3) follows.

Consider inequality $(5.4)$ and rewrite $w(\cdot, 0)=: w_{0}$ from $(5.2)$ as

$$
w_{0}=\mathcal{S}_{\tilde{a}_{0} \tilde{p}_{0} \tilde{\gamma}_{0}}^{-1}\left(\psi_{0}-\eta_{\sigma_{0}}\right)+\mathcal{S}_{\tilde{a}_{0} \tilde{p}_{0} \tilde{\gamma}_{0}}^{-1}\left(\eta_{\sigma_{0}}-\eta_{\tilde{\sigma}_{0}}\right) .
$$

To estimate this, we first estimate the linear operator $\mathcal{S}_{a p \gamma}^{-1}$ :

$$
\left\|\mathcal{S}_{\text {ap }}^{-1} \psi\right\|_{\mathrm{H}_{1}} \leq 2\left(1+|p|^{2}\right)^{1 / 2}\|\psi\|_{\mathrm{H}_{1}} .
$$

The first term in (5.7) is in the appropriate form, for the second term we recall that $\eta$ is a $\mathrm{C}^{1}$-map. Thus for some $\theta_{2} \in[0,1]$

$$
\eta_{\tilde{\sigma}_{0}}-\eta_{\sigma_{0}}=\left.\sum_{j=1}^{2 d+2}\left(\tilde{\sigma}_{0}-\sigma_{0}\right)_{j} \partial_{\sigma_{j}} \eta_{\sigma}\right|_{\sigma=\theta_{1} \tilde{\sigma}_{0}+\left(1-\theta_{2}\right) \sigma_{0}} .
$$


To calculate the norm of this expression, note that

$$
\partial_{\sigma} \eta_{\sigma}=\mathcal{S}_{a p \gamma} z_{\mu, p}, \quad \text { where } z_{\mu, p}:=\left\{\mathrm{i} p \eta_{\mu}+\nabla \eta_{\mu}, \mathrm{i} x \eta_{\mu}, \mathrm{i} \eta_{\mu}, \partial_{\mu} \eta_{\mu}\right\}
$$

and $\left\|z_{\mu, p}\right\|_{\mathrm{H}_{1}} \leq \sqrt{5} C_{I}\left(1+|p|^{2}\right)^{1 / 2}$. Let $n\left(\sigma, \sigma_{0}\right):=\left(\sigma-\sigma_{0}\right) \theta_{2}+\sigma_{0}$, and define $g^{2}:=1+\left|\tilde{p}_{0}-p_{0}\right|^{2}+p_{0}^{2}$. The $\mathrm{H}_{1}$-norm of (5.9), using (5.8) and (5.10) is

$$
\begin{aligned}
\left\|\eta_{\tilde{\sigma}_{0}}-\eta_{\sigma_{0}}\right\|_{\mathrm{H}_{1}} & \leq\left|\tilde{\sigma}_{0}-\sigma_{0}\right||| \partial_{\sigma} \eta_{\sigma} \|\left._{\mathrm{H}_{1}}\right|_{\sigma=n\left(\tilde{\sigma}_{0}, \sigma_{0}\right)} \\
& \leq\left. 2 \sqrt{5} C_{I}\left(1+|p|^{2}\right)\right|_{p=n\left(\tilde{p}_{0}, p_{0}\right)}\left|\tilde{\sigma}_{0}-\sigma_{0}\right| \leq 9 C_{I} g^{2}\left|\tilde{\sigma}_{0}-\sigma_{0}\right| .
\end{aligned}
$$

We now calculate the $\mathrm{H}_{1}$ norm of $w_{0}$ (see (5.7)) using (5.3), (5.8) with momentum $p=\tilde{p}_{0}-p_{0}+p_{0}$ and (5.11). We find

$$
\begin{aligned}
\left\|w_{0}\right\|_{\mathrm{H}_{1}} & \leq 2 g\left(\left\|\psi_{0}-\eta_{\sigma_{0}}\right\|_{\mathrm{H}_{1}}+\left\|\eta_{\tilde{\sigma}_{0}}-\eta_{\sigma_{0}}\right\|_{\mathrm{H}_{1}}\right) \\
& \leq 2 g\left(1+9 C_{I} c_{I} g^{2}\right)\left\|\psi_{0}-\eta_{\sigma_{0}}\right\|_{\mathrm{H}_{1}} .
\end{aligned}
$$

The coefficient above is less then $c g^{4}+C$, and $g^{4} \leq 3\left(1+c_{I}^{4}\left\|\psi_{0}-\eta_{\sigma_{0}}\right\|_{\mathrm{L}^{2}}^{4}+\left|p_{0}\right|^{4}\right)$. Inserting and simplifying gives the inequality (5.4).

The quantity appearing in the third and last inequality (5.5), can be rewritten as

$$
\left\langle\epsilon_{V} x\right\rangle^{r / 2} w_{0}=\left\langle\epsilon_{V} x\right\rangle^{r / 2} \mathcal{S}_{\tilde{a}_{0} \tilde{p}_{0} \tilde{\gamma}_{0}}^{-1}\left(\left(\psi_{0}-\eta_{\sigma_{0}}\right)+\left(\eta_{\sigma_{0}}-\eta_{\tilde{\sigma}_{0}}\right)\right)
$$

We begin our calculation of the norm of (5.13) by considering the linear operator $\left\langle\epsilon_{V} x\right\rangle^{r / 2} \mathcal{S}_{a p \gamma}$. We have

$$
\left\langle\epsilon_{V} x\right\rangle^{r / 2} \mathcal{S}_{a p \gamma} \psi=\mathcal{S}_{a p \gamma}\left\langle\epsilon_{V}(x-a)\right\rangle^{r / 2} \psi
$$

and $\left\|\mathcal{S}_{a p \gamma} \psi\right\|_{\mathrm{L}^{2}}=\|\psi\|_{\mathrm{L}^{2}}$. From Lemma A.4 we obtain

$$
\begin{aligned}
\left\|\left\langle\epsilon_{V} x\right\rangle^{r / 2} \mathcal{S}_{a p \gamma} \psi\right\|_{\mathrm{L}^{2}} & \leq\left\|\left\langle\epsilon_{V}\left(x-\left(a-a_{0}\right)-a_{0}\right)\right\rangle^{r / 2} \psi\right\|_{\mathrm{L}^{2}} \\
& \leq 3^{\max (r / 2, r-1)}\left(\left\|\left\langle\epsilon_{V} x\right\rangle^{r / 2} \psi\right\|_{\mathrm{L}^{2}}+g_{2}\|\psi\|_{\mathrm{L}^{2}}\right),
\end{aligned}
$$

where $\left.g_{2}:=\left(\epsilon_{V}\left|a-a_{0}\right|\right)^{r / 2}+\left(\epsilon_{V}\left|a_{0}\right|\right)^{r / 2}\right)$. Using this we find the $\mathrm{L}^{2}$-norm of $(5.13)$ to be

$$
\begin{aligned}
\left\|\left\langle\epsilon_{V} x\right\rangle^{r / 2} w_{0}\right\|_{\mathrm{L}^{2}} \leq C\left(\left\|\left\langle\epsilon_{V} x\right\rangle^{r / 2}\left(\psi_{0}-\eta_{\sigma_{0}}\right)\right\|_{\mathrm{L}^{2}}+g_{2}\left\|\psi_{0}-\eta_{\sigma_{0}}\right\|_{\mathrm{L}^{2}}\right. \\
\left.+\left\|\left\langle\epsilon_{V} x\right\rangle^{r / 2}\left(\eta_{\tilde{\sigma}_{0}}-\eta_{\sigma_{0}}\right)\right\|_{\mathrm{L}^{2}}+g_{2}\left\|\eta_{\tilde{\sigma}_{0}}-\eta_{\sigma_{0}}\right\|_{\mathrm{L}^{2}}\right) .
\end{aligned}
$$

The first and second term of the above expression is in an appropriate form. We bound the third term by using (5.9), (5.10) and (5.14) to get

$$
\begin{aligned}
\left\|\left\langle\epsilon_{V} x\right\rangle^{r / 2}\left(\eta_{\tilde{\sigma}_{0}}-\eta_{\sigma_{0}}\right)\right\|_{\mathrm{L}^{2}} & \leq\left.\left|\tilde{\sigma}_{0}-\sigma_{0}\right|\left\|\left\langle\epsilon_{V}(x-a)\right\rangle^{r / 2} z_{p, \mu}\right\|_{\mathrm{L}^{2}}\right|_{\sigma=n\left(\tilde{\sigma}_{0}, \sigma_{0}\right)} \\
& \leq 3^{\max (r / 2, r-1)} \sqrt{5} C_{I} g\left(1+g_{2}\right)\left|\tilde{\sigma}_{0}-\sigma_{0}\right| .
\end{aligned}
$$

The last term of (5.16) is straight forward to bound:

$$
\begin{aligned}
\left\|\eta_{\tilde{\sigma}_{0}}-\eta_{\sigma_{0}}\right\|_{\mathrm{L}^{2}} & \leq\left.\left|\tilde{\sigma}_{0}-\sigma_{0}\right|\left\|\partial_{\sigma} \eta_{\sigma}\right\|_{\mathrm{L}^{2}}\right|_{\sigma=n\left(\tilde{\sigma}_{0}, \sigma_{0}\right)} \\
& \leq\left.\left|\tilde{\sigma}_{0}-\sigma_{0}\right|\left\|z_{p, \mu}\right\|_{\mathrm{L}^{2}}\right|_{p=n\left(\tilde{p}_{0}, p_{0}\right) \mu=n\left(\tilde{\mu}_{0}, \mu_{0}\right)} \leq \sqrt{5} C_{I} g\left|\tilde{\sigma}_{0}-\sigma_{0}\right| .
\end{aligned}
$$


Inserting (5.17) and (5.18) into (5.16) gives

$$
\begin{aligned}
\left\|\left\langle\epsilon_{V} x\right\rangle^{r / 2} w_{0}\right\|_{\mathrm{L}^{2}} \leq C\left(\|\left\langle\epsilon_{V} x\right\rangle^{r / 2}\left(\psi_{0}-\right.\right. & \left.\eta_{\sigma_{0}}\right) \|_{\mathrm{L}^{2}} \\
& \left.+\left(g_{2}+g\left(1+2 g_{2}\right)\right)\left\|\psi_{0}-\eta_{\sigma_{0}}\right\|_{\mathrm{L}^{2}}\right)
\end{aligned}
$$

where $C$ depend only on $C_{I}, c_{I}$ and $r$. We simplify this, by repeatedly using Cauchy's inequality and (5.3) on the expression in front of the $\left\|\psi_{0}-\eta_{\sigma_{0}}\right\|_{\mathrm{L}^{2}}$-term, to obtain

$$
\begin{aligned}
\left\|\left\langle\epsilon_{V} x\right\rangle^{r / 2} w_{0}\right\|_{\mathrm{L}^{2}} \leq & C\left(\left\|\left\langle\epsilon_{V} x\right\rangle^{r / 2}\left(\psi_{0}-\eta_{\sigma_{0}}\right)\right\|_{\mathrm{L}^{2}}+\left(1+\left\|\psi_{0}-\eta_{\sigma_{0}}\right\|_{\mathrm{L}^{2}}^{2}\right.\right. \\
& \left.\left.+\epsilon_{V}^{r}\left\|\psi_{0}-\eta_{\sigma_{0}}\right\|_{\mathrm{L}^{2}}^{r}+\left|p_{2}\right|^{2}+\left(\epsilon_{V}\left|a_{0}\right|\right)^{r}\right)\left\|\psi_{0}-\eta_{\sigma_{0}}\right\|_{\mathrm{L}^{2}}\right) .
\end{aligned}
$$

This gives the third inequality of the proposition.

Recall the initial energy bound (2.22)

$$
\left\|\psi_{0}-\eta_{\sigma_{0}}\right\|_{\mathrm{H}_{1}}+\left\|\left\langle\epsilon_{V} x\right\rangle^{r / 2}\left(\psi_{0}-\eta_{\sigma_{0}}\right)\right\|_{\mathrm{L}^{2}} \leq \epsilon_{0},
$$

and the bound on the initial kinetic and potential energy for the solitary wave $(2.23)$

$$
\frac{1}{2}\left(p_{0}^{2}+V\left(a_{0}\right)\right) \leq \epsilon_{h}
$$

We have the corollary

Corollary 5.2. Let (2.22), (2.23) and (2.4)-(2.6) hold with $\epsilon_{0}<\delta$. Then

$$
\begin{gathered}
\left|\tilde{\sigma}_{0}-\sigma_{0}\right| \leq c_{I} \epsilon_{0}, \quad\left\|w_{0}\right\|_{\mathrm{H}_{1}} \leq C_{1} \epsilon_{0}, \\
\left\|\left\langle\epsilon_{V} x\right\rangle^{r / 2} w_{0}\right\|_{\mathrm{L}^{2}} \leq C_{2} \epsilon_{0}
\end{gathered}
$$

and

$$
h\left(\tilde{a}_{0}, \tilde{p}_{0}\right) \leq C_{3}\left(\epsilon_{h}+\epsilon_{0}^{2}+\epsilon_{V} \epsilon_{0}\right),
$$

where $C_{1}, C_{2}$ and $C_{3}$ depend only on $c_{L}, c_{V}(E q .(2.6)), C_{E}:=\max \left(\epsilon_{V}, \epsilon_{0}, \epsilon_{h}\right)$ and the constants in Proposition 5.1.

Proof. Starting from Proposition 5.1 the first three inequalities follow directly through the energy bounds $(2.22),(2.23)$ together with the observation that either $\epsilon_{V}\left|a_{0}\right| \leq c_{L}$ or $c_{V}\left(\epsilon_{V}\left|a_{0}\right|\right)^{r} \leq V\left(a_{0}\right) \leq 2 \epsilon_{h}$. We also use that $\epsilon_{h}, \epsilon_{0}$ and $\epsilon_{V}$ are all bounded by a constant $C_{E}$.

The last inequality follows from the fact that $h(a, p):=\left(p^{2}+V(a)\right) / 2$ is a $\mathrm{C}^{1}$ function. For some $\theta \in[0,1]$

$$
\begin{aligned}
h(a, p)-h\left(a_{0}, p_{0}\right)= & \left(\left(p-p_{0}\right) \theta+p_{0}\right) \cdot\left(p-p_{0}\right) \\
& +\frac{1}{2}\left(a-a_{0}\right) \cdot \nabla V\left(\left(a-a_{0}\right) \theta+a_{0}\right) .
\end{aligned}
$$


Thus, using (2.4), and $\langle x+y\rangle^{r-1} \leq 3^{\max (0,(r-3) / 2)}\left(1+2^{(r-1) / 2}\left(|x|^{r-1}+|y|^{r-1}\right)\right)$ gives

$$
\begin{aligned}
\left|h(a, p)-h\left(a_{0}, p_{0}\right)\right| \leq C & \left(\left|p-p_{0}\right|^{2}+\left|p_{0}\right|^{2}+\right. \\
& \left.\epsilon_{V}^{2}\left|a-a_{0}\right|\left(1+\left|\epsilon_{V}\left(a-a_{0}\right)\right|^{r-1}+\left|\epsilon_{V} a_{0}\right|^{r-1}\right)\right) .
\end{aligned}
$$

With $p=\tilde{p}_{0}$ and $a=\tilde{a}_{0}$ above, and $\left|\tilde{\sigma}_{0}-\sigma_{0}\right| \leq c_{I} \epsilon_{0}, h\left(a_{0}, p_{0}\right) \leq \epsilon_{h},(2.23)$ and (2.6) we have have shown (5.25).

\section{Bounds on soliton position and momentum}

In this section we use the bounded initial soliton energy, Corollary 5.2, to find upper bounds on position and momentum of the solitary wave. We express the norms first in terms of $h\left(\tilde{a}_{0}, \tilde{p}_{0}\right)$ and the small parameters. In Corollary 6.2 we state the final result, where the bounds are just constants times the small parameters $\epsilon_{0}, \epsilon_{h}$ and $\epsilon_{V}$.

Recall (see (2.4) and (2.6)) that the potential $V$ is non-negative and satisfies the following upper and lower bounds:

$$
\left|\partial_{x}^{\beta} V\right| \leq C_{V} \epsilon_{V}\left\langle\epsilon_{V} a\right\rangle^{r-1}, \quad \text { for }|\beta|=1,
$$

and, if $\epsilon_{V}|a| \geq c_{L}$ then

$$
V(a) \geq c_{V}\left(\epsilon_{V}|a|\right)^{r} .
$$

To obtain the desired estimates on $a$ and $p$ we will use the fact that the soliton energy,

$$
h(a, p):=\frac{1}{2}\left(p^{2}+V(a)\right),
$$

is essentially conserved. We abbreviate $\alpha:=\left\{\alpha^{\mathrm{tr}}, \alpha^{\mathrm{b}}, \alpha_{2 d+1}, \alpha_{2 d+2}\right\}$. The size of $\alpha$ is measured by $|\alpha|^{2}:=\sum_{j}\left|\alpha_{j}\right|^{2}$ and $|\alpha|_{\infty}:=\sup _{s \leq t}|\alpha(s)|$. We have the following:

Proposition 6.1. Let $V$ satisfy conditions (6.1) and (6.2). Let $h_{0}:=h\left(\tilde{a}_{0}, \tilde{p}_{0}\right)$, and set

$$
\tilde{T}_{1}:=\frac{C_{T_{1}}}{\left(\epsilon_{V}^{2}+|\alpha|_{\infty}\right)\left(1+\epsilon_{V}+h_{0}\right)}, \quad C_{\tilde{T}_{1}}:=\frac{c_{V}}{2^{\max (2, r-1) / 2} C_{V} d},
$$

where the constants $C_{V}$ and $c_{V}$ are related to the growth rate of the potential (see (2.4) and (2.6)). Then for times $t \leq \tilde{T}_{1}$ :

$$
|p| \leq C_{\tilde{p}}\left(\sqrt{h_{0}}+|\alpha|_{\infty} t+\epsilon_{V}\right) \quad \text { and } \quad \epsilon_{V}|a| \leq C_{a},
$$

where $C_{a}$ and $C_{\tilde{p}}$ depend only on $c_{L}, c_{V}, C_{\tilde{T}_{1}}, r, d, C_{3}$ and $C_{E}=\max \left(\epsilon_{V}, \epsilon_{0}, \epsilon_{h}\right)$. $C_{3}$ is the constant in Corollary 5.2 .

Proof. First we estimate $p$ in terms of $a$, using the almost conservation of $h(a, p)$

$$
\frac{d}{d t} h(a, p)=\frac{1}{2}(2 p \cdot(\dot{p}+\nabla V(a))+\nabla V(a) \cdot(\dot{a}-2 p)) .
$$


Now recall the definitions $\alpha^{\mathrm{b}}:=-\dot{p}-\nabla V(a)$ and $\alpha^{\mathrm{tr}}:=\dot{a}-2 p$ together with the upper bound (6.1) of the potential $|\nabla V| \leq d^{1 / 2} C_{V} \epsilon_{V}\left\langle\epsilon_{V} a\right\rangle^{r-1}$ to obtain

$$
\left|\mathrm{d}_{t} h(a, p)\right| \leq|\alpha||p|+\frac{1}{2} C_{V} d^{1 / 2} \epsilon_{V}|\alpha|\left\langle\epsilon_{V} a\right\rangle^{r-1} .
$$

Integration in time and simplification gives

$$
h(a(t), p(t)) \leq h_{0}+t\left(|\alpha|_{\infty}\right)\left(|p|_{\infty}+2^{-1} d^{1 / 2} C_{V} \epsilon_{V}\left\langle\epsilon_{V}|a|_{\infty}\right\rangle^{r-1}\right) .
$$

Recall that $h=2^{-1}\left(p^{2}+V(a)\right)$ and that $V \geq 0$, thus $|p|^{2} \leq 2 h$. Solving the resulting quadratic inequality for $|p|_{\infty}>0$ we find that

$$
|p|_{\infty} \leq \sqrt{2 h_{0}}+3 t|\alpha|_{\infty}+2^{-1} d^{1 / 2} C_{V} \epsilon_{V}\left\langle\epsilon_{V}|a|_{\infty}\right\rangle^{r-1}
$$

The Eqn. (6.8) also implies

$$
\sup _{s \leq t} V(a(s)) \leq 2 h_{0}+2 t|\alpha|_{\infty}\left(|p|_{\infty}+2^{-1} d^{1 / 2} C_{V} \epsilon_{V}\left\langle\epsilon_{V}|a|_{\infty}\right\rangle^{r-1}\right) .
$$

As can be seen in (6.9) we need to consider the possibility of large $\epsilon_{V}|a|$. Let $\epsilon_{V}|a| \geq c_{L}$, with $c_{L}$ as in (6.2) then $V(a) \geq c_{V}\left(\epsilon_{V}|a|\right)^{r}$. Inserting this lower bound and (6.9) into (6.10) we obtain

$$
c_{V}\left(\epsilon_{V}|a|_{\infty}\right)^{r} \leq 2 h_{0}+2 t|\alpha|_{\infty}\left(\sqrt{2 h_{0}}+3 t|\alpha|_{\infty}+d^{1 / 2} C_{V} \epsilon_{V}\left\langle\epsilon_{V}|a|_{\infty}\right\rangle^{r-1}\right) .
$$

Lemma A.4 shows $\left\langle\epsilon_{V}|a|_{\infty}\right\rangle^{r-1} \leq 2^{\max (0, r-3) / 2}\left(1+\left(\epsilon_{V}|a|_{\infty}\right)^{r-1}\right)$ for $r \geq 1$. If the maximal time satisfies the inequality $t \leq \tilde{T}_{1}$ (see (6.4)), then the above inequality implies

$$
\epsilon_{V}|a|_{\infty} \leq\left(\frac{2}{c_{V}}\left(C_{4}+2 C_{\tilde{T}_{1}}+6 C_{\tilde{T}_{1}}^{2}+\frac{1}{2} c_{V}\right)^{1 / r}=: \tilde{C}_{a}\right.
$$

where we have used that $h_{0}$ is bounded by the constant $C_{E}$. Thus, either $\epsilon_{V}|a| \leq c_{L}$ holds or, for the given time interval, (6.12) holds. In both cases $\epsilon_{V}|a| \leq C_{a}$, where the constant only depends on $C_{4}=C_{3} C_{E}, C_{\tilde{T}_{1}}, c_{V}, c_{L}$ and $r$. We insert this upper bound on $\epsilon_{V}|a|$ into (6.9) and for times $t \leq \tilde{T}_{1}$ we find

where $C_{\tilde{p}}:=3+d^{1 / 2} C_{V} C_{\tilde{a}}^{r-1}$.

$$
|p|_{\infty} \leq C_{\tilde{p}}\left(\sqrt{h_{0}}+|\alpha|_{\infty} t+\epsilon_{V}\right),
$$

Using the Corollary 5.2 we express the above proposition in terms of $\epsilon_{h}$ rather than $h_{0}$. Recall the requirement on $\delta$ from Theorem 4.1

Corollary 6.2. Let $V$ satisfy (2.4)-(2.6) and let $\psi_{0} \in U_{\delta} \cap \mathscr{H}_{1, r}$. Furthermore, let $\psi_{0}$ satisfy the $\epsilon_{0}$-energy bound (2.22) for $\eta_{\sigma_{0}}$ with $\sigma_{0}=\left\{a_{0}, p_{0}, \gamma_{0}, \mu_{0}\right\}$, and let $h\left(a_{0}, p_{0}\right) \leq \epsilon_{h}$ (i.e., $\left.(2.23)\right)$. Let

$$
T_{1}:=\frac{C_{T_{1}}}{\left(\epsilon_{V}^{2}+|\alpha|_{\infty}\right)\left(1+\epsilon_{V}+\epsilon_{h}+\epsilon_{V}\right)}, \quad T_{2}:=\frac{\sqrt{\epsilon_{h}}}{|\alpha|_{\infty}+\epsilon_{V}^{2}},
$$

where

$$
C_{T_{1}}:=\frac{C_{\tilde{T}_{1}}}{\left(1+C_{3}\right)\left(1+C_{E}^{2}\right)} .
$$


Then for times $t \leq \min \left(T_{1}, T_{2}\right)$ :

$$
|p| \leq C_{p}\left(\sqrt{\epsilon_{h}}+\epsilon_{0}+\epsilon_{V}\right) \quad \text { and } \quad \epsilon_{V}|a| \leq C_{a}
$$

where $C_{p}$ depends on $C_{E}=\max \left(\epsilon_{V}, \epsilon_{0}, \epsilon_{h}\right), C_{V}, d, r$ and $C_{a} . C_{3}$ is defined in Corollary 5.2 and $C_{a}$ in Proposition 6.1. The constant $C_{V}$ is defined in (2.4).

Proof. Under the assumptions of the corollary we have that Corollary 5.2 holds and hence

$$
h\left(\tilde{a}_{0}, \tilde{p}_{0}\right) \leq C_{3}\left(\epsilon_{h}+\epsilon_{0}^{2}+\epsilon_{V} \epsilon_{0}\right) .
$$

We now modify the constants and estimates of Proposition 6.1 to take the upper bound of $h_{0}$ into account. The new, maximal time derived from $\tilde{T}_{1}$ becomes $T_{1} \leq \tilde{T}_{1}$. For times shorter than this time, $t \leq T_{1}$, the bound on $\epsilon_{V}|a|$ remains the same. Using this estimate for $\epsilon_{V}|a|$, we simplify the $|p|$ estimate. Note first that $\sqrt{h_{0}}+\epsilon_{V} \leq\left(\sqrt{\epsilon_{h}}+\epsilon_{0}+\epsilon_{V}\right)\left(1+2 \sqrt{C_{3}}\right)$, inserted into (6.5) gives

$$
|p| \leq \frac{1}{2} C_{p}\left(\sqrt{\epsilon_{h}}+\epsilon_{V}+\epsilon_{0}+|\alpha| t\right),
$$

where $C_{p}$ depends on $C_{3}, C_{E}, C_{a}$ and $d$ and $r$. With the choice of time interval $T_{2}$ such that $t \leq T_{2}$, where $T_{2}$ is given in (6.14), we obtain $|p| \leq C_{p}\left(\sqrt{\epsilon_{h}}+\epsilon_{0}+\epsilon_{V}\right)$.

\section{Lyapunov functional}

In this section we define the Lyapunov functional and calculate its time derivative in the moving frame. Recall the definition of $\mathcal{E}_{\mu}(\psi)$ in $(1.20)$ together with decomposition (4.4): $\psi=\mathcal{S}_{a p \gamma}\left(\eta_{\mu}+w\right)$, with $w \perp J \mathrm{~T}_{\eta} \mathrm{M}_{\mathrm{s}}$. Define the Lyapunov functional, $\Lambda$, as

$$
\Lambda:=\mathcal{E}_{\mu}\left(\eta_{\mu}+w\right)+\frac{1}{2}\left\langle\mathcal{R}_{V}\left(\eta_{\mu}+w\right), \eta_{\mu}+w\right\rangle-\mathcal{E}_{\mu}\left(\eta_{\mu}\right)-\frac{1}{2}\left\langle\mathcal{R}_{V} \eta_{\mu}, \eta_{\mu}\right\rangle
$$

Here we show that the Lyapunov functional $\Lambda$ is an almost conserved quantity. We begin by computing its time derivative. Let $\alpha^{\mathrm{b}}:=-\dot{p}-\nabla V(a)$ and $\alpha^{\mathrm{tr}}:=\dot{a}-2 p$ (boost and translation coefficients). We have the following proposition

Proposition 7.1. Given a solution $\psi \in \mathscr{H}_{1, r} \cap U_{\delta}$ to (1.1), define $\eta_{\mu}$ and $w$ as above. Then

$$
\frac{d}{d t} \Lambda=p \cdot\left\langle\nabla_{a} \mathcal{R}_{V} w, w\right\rangle-\alpha^{\mathrm{tr}} \cdot \mathrm{D}^{2} V(a) \cdot\langle x w, w\rangle+R
$$

where

$$
\begin{aligned}
R:= & \alpha^{\mathrm{b}} \cdot\langle\mathrm{i} w, \nabla w\rangle+2 p \cdot\left\langle\nabla_{a} \mathcal{R}_{V} \eta_{\mu}, w\right\rangle-\frac{1}{2} \alpha^{\mathrm{tr}} \cdot\left\langle\nabla_{a} \mathcal{R}_{V} \eta_{\mu}, \eta_{\mu}\right\rangle \\
& +\frac{\dot{\mu}}{2}\|w\|_{\mathrm{L}^{2}}^{2}-\dot{\mu}\left\langle\mathcal{R}_{V} \eta_{\mu}, \partial_{\mu} \eta_{\mu}\right\rangle .
\end{aligned}
$$


Before proceeding to the proof, we recall the definition of the moving frame solution $u$ defined by

$$
u(x, t):=\mathrm{e}^{-\mathrm{i} p \cdot x-\mathrm{i} \gamma} \psi(x+a, t) .
$$

Here $a, p$ and $\gamma$ depend on time, in a way determined by the decomposition of Section 4, and the function $\psi$ is a solution of the nonlinear Schrödinger equation (1.1). In the moving frame the Lyapunov functional $\Lambda$ takes the form

$$
\Lambda=\mathcal{E}_{\mu}(u)+\frac{1}{2}\left\langle\mathcal{R}_{V} u, u\right\rangle-\mathcal{E}_{\mu}\left(\eta_{\mu}\right)-\frac{1}{2}\left\langle\mathcal{R}_{V} \eta_{\mu}, \eta_{\mu}\right\rangle
$$

We begin with some auxiliary lemmas.

Lemma 7.2. Let $\psi \in \mathscr{H}_{1, r}$ be a solution to (1.1). Then

$$
\partial_{t}\langle\psi,-\mathrm{i} \nabla \psi\rangle=-\langle(\nabla V) \psi, \psi\rangle \quad \text { and } \quad \partial_{t}\langle x \psi, \psi\rangle=2\langle\psi,-\mathrm{i} \nabla \psi\rangle .
$$

Proof. The first part of this lemma was proved in [17]. To prove the second part we use the equation

$$
\partial_{t}\left(x_{k}|\psi|^{2}\right)=\mathrm{i} \nabla \cdot\left(x_{k} \bar{\psi} \nabla \psi-x_{k} \psi \nabla \bar{\psi}\right)-\mathrm{i}\left(\bar{\psi} \partial_{k} \psi-\psi \partial_{k} \bar{\psi}\right)
$$

understood in a weak sense, which follows from the nonlinear Schrödinger equation (1.1). Formally, integrating this equation and using that the divergence term vanishes gives the second equation in (7.6). To do this rigorously, let $\chi$ be a $\mathrm{C}^{1}$ function such that $|\nabla \chi(x)| \leq C$ and

$$
\chi(x):= \begin{cases}1 & |x| \leq 1 \\ 0 & |x|>2\end{cases}
$$

and let $\chi_{R}(x):=\chi\left(\frac{x}{R}\right)$. Abbreviate $j_{k}:=\left(x_{k} \bar{\psi} \nabla \psi-x_{k} \psi \nabla \bar{\psi}\right)$ and let $R>1$. We multiply the divergence term by $\chi_{R}$. Integration by parts gives

$$
\left|\int\left(\nabla \cdot j_{k}\right) \chi_{R} \mathrm{~d}^{d} x\right|=\left|\int j_{k} \cdot \nabla \chi_{R}(x) \mathrm{d}^{d} x\right| \leq \frac{C}{R} \int\left|j_{k}\right| \mathrm{d}^{d} x .
$$

We note that $j_{k} \in \mathrm{L}^{1}$ for all $k$, and is independent of $R$, thus as $R \rightarrow \infty$, this term vanishes. The remaining terms give in the limit $R \rightarrow \infty$ the second equation in (7.6).

Lemma 7.3. Let $\psi \in \mathscr{H}_{1, r}$ be a solution to (1.1), and let $u$ be defined as above. Then

$$
\begin{aligned}
\frac{d}{d t}\left(\mathcal{E}_{\mu}(u)+\frac{1}{2}\left\langle\mathcal{R}_{V} u, u\right\rangle\right)= & p \cdot\left\langle\nabla_{a} \mathcal{R}_{V} u, u\right\rangle-\frac{1}{2} \alpha^{\mathrm{tr}} \cdot \mathrm{D}^{2} V(a) \cdot\langle x u, u\rangle \\
& +\frac{1}{2} \dot{\mu}\|u\|_{\mathrm{L}^{2}}^{2}+\alpha^{\mathrm{b}} \cdot\langle\mathrm{i} u, \nabla u\rangle,
\end{aligned}
$$

where $\alpha^{\text {tr }}:=\dot{a}-2 p$ and $\alpha^{\mathrm{b}}=-\dot{p}-\nabla V(a)$. 
Proof. The functional $\mathcal{E}_{\mu}(u)+\frac{1}{2}\left\langle\mathcal{R}_{V} u, u\right\rangle$, is related to the Hamiltonian functional by

$$
\begin{aligned}
\mathcal{E}_{\mu}(u)+\frac{1}{2}\left\langle\mathcal{R}_{V} u, u\right\rangle= & \mathcal{H}_{V}(\psi)+\frac{1}{2}\left(p^{2}+\mu\right)\|\psi\|_{\mathrm{L}^{2}}^{2}-p \cdot\langle\mathrm{i} \psi, \nabla \psi\rangle \\
& -\frac{1}{2} \int(V(a)+\nabla V(a) \cdot(x-a))|\psi|^{2} \mathrm{~d}^{d} x,
\end{aligned}
$$

which is obtained by substituting (7.4) into $\mathcal{E}_{\mu}(u)+\frac{1}{2}\left\langle\mathcal{R}_{V} u, u\right\rangle$. Using the facts that the mass $\|\psi\|_{\mathrm{L}^{2}}^{2}$ and Hamiltonian $\mathcal{H}_{V}(\psi)$ are time independent, together with the Ehrenfest relations, Lemma 7.2, we obtain

$$
\begin{aligned}
\frac{d}{d t}\left(\mathcal{E}_{\mu}(u)+\frac{1}{2}\left\langle\mathcal{R}_{V} u, u\right\rangle\right)= & \left(\frac{\dot{\mu}}{2}+p \cdot \dot{p}\right)\|\psi\|_{\mathrm{L}^{2}}^{2}-\dot{p} \cdot\langle\mathrm{i} \psi, \nabla \psi\rangle+p \cdot\langle(\nabla V) \psi, \psi\rangle \\
& -\frac{\dot{a}}{2} \cdot \mathrm{D}^{2} V(a) \cdot \int(x-a)|\psi|^{2} \mathrm{~d}^{d} x-\nabla V(a) \cdot\langle\mathrm{i} \psi, \nabla \psi\rangle .
\end{aligned}
$$

Collecting $p \cdot \dot{p}$ and $p \cdot \nabla V$ together, and combining $\dot{p}$ and $\nabla V(a)$ gives

$$
\begin{aligned}
\frac{d}{d t}\left(\mathcal{E}_{\mu}(u)+\right. & \left.\frac{1}{2}\left\langle\mathcal{R}_{V} u, u\right\rangle\right)=\frac{\dot{\mu}}{2}\|\psi\|_{\mathrm{L}^{2}}^{2}+p \cdot\langle(\dot{p}+\nabla V) \psi, \psi\rangle \\
& -(\dot{p}+\nabla V(a)) \cdot\langle\mathrm{i} \psi, \nabla \psi\rangle-\frac{1}{2} \dot{a} \cdot \mathrm{D}^{2} V(a) \cdot \int(x-a)|\psi|^{2} \mathrm{~d}^{d} x
\end{aligned}
$$

From the definition of $u,(7.4)$, the following relations hold

$$
\begin{gathered}
\|\psi\|_{\mathrm{L}^{2}}=\|u\|_{\mathrm{L}^{2}}, \quad\langle\mathrm{i} \psi, \nabla \psi\rangle=p\|u\|_{\mathrm{L}^{2}}^{2}+\langle\mathrm{i} u, \nabla u\rangle, \\
\langle(\nabla V) \psi, \psi\rangle=\left\langle\left(\nabla V_{a}\right) u, u\right\rangle, \quad\langle(x-a) \psi, \psi\rangle=\langle x u, u\rangle .
\end{gathered}
$$

Substitution of (7.13)-(7.14) into (7.12) gives, after cancellation of the $p \cdot \dot{p}$ terms,

$$
\begin{aligned}
& \frac{d}{d t}\left(\mathcal{E}_{\mu}(u)+\frac{1}{2}\left\langle\mathcal{R}_{V} u, u\right\rangle\right)=\frac{\dot{\mu}}{2}\|u\|_{\mathrm{L}^{2}}^{2}+p \cdot\left\langle\left(\nabla V_{a}-\nabla V(a)\right) u, u\right\rangle \\
&-(\dot{p}+\nabla V(a)) \cdot\langle\mathrm{i} u, \nabla u\rangle-\frac{1}{2} \dot{a} \cdot \mathrm{D}^{2} V(a) \cdot \int x|u|^{2} \mathrm{~d}^{d} x .
\end{aligned}
$$

The last remaining step is to rewrite the second last term as $\dot{a}-2 p+2 p$ and combine its $p$ term with the difference of the potentials, recalling the definition of $\mathcal{R}_{V}$, to obtain

$$
\begin{aligned}
\frac{d}{d t}\left(\mathcal{E}_{\mu}(u)+\right. & \left.\frac{1}{2}\left\langle\mathcal{R}_{V} u, u\right\rangle\right)=\frac{\dot{\mu}}{2}\|u\|_{\mathrm{L}^{2}}^{2}+p \cdot\left\langle\left(\nabla_{a} \mathcal{R}_{V}\right) u, u\right\rangle \\
& -(\dot{p}+\nabla V(a)) \cdot\langle\mathrm{i} u, \nabla u\rangle+\frac{1}{2}(2 p-\dot{a}) \cdot \mathrm{D}^{2} V(a) \cdot \int x|u|^{2} \mathrm{~d}^{d} x
\end{aligned}
$$

Identification of the boost coefficient $\alpha^{\mathrm{b}}:=-\dot{p}-\nabla V(a)$ and the translation coefficient $\alpha^{\text {tr }}:=\dot{a}-2 p$ gives the lemma.

The time derivative of the second part of the Lyapunov functional (7.5) is computed in the next lemma. 
Lemma 7.4. Let $\eta_{\mu}$ be the solution of (2.18), and let $\mu$ depend on $t$. Then

$$
\begin{aligned}
\frac{d}{d t}\left(\mathcal{E}_{\mu}\left(\eta_{\mu}\right)+\right. & \left.\frac{1}{2}\left\langle\mathcal{R}_{V} \eta_{\mu}, \eta_{\mu}\right\rangle\right)= \\
& \frac{\dot{\mu}}{2}\left\|\eta_{\mu}\right\|_{\mathrm{L}^{2}}^{2}+\left(p+\frac{1}{2} \alpha^{\mathrm{tr}}\right) \cdot\left\langle\nabla_{a} \mathcal{R}_{V} \eta_{\mu}, \eta_{\mu}\right\rangle+\dot{\mu}\left\langle\mathcal{R}_{V} \eta_{\mu}, \partial_{\mu} \eta_{\mu}\right\rangle
\end{aligned}
$$

where $\alpha^{\mathrm{tr}}:=\dot{a}-2 p$.

Proof. The result follows directly, upon recalling that $\mathcal{E}_{\mu}^{\prime}\left(\eta_{\mu}\right)=0$ and $\frac{1}{2} \alpha^{\operatorname{tr}}+p$ $=\frac{\dot{a}}{2}$.

To proceed to the proof of Proposition 7.1, we restate our condition for unique decomposition of the solution to the nonlinear Schrödinger equation, $\psi \in U_{\delta} \cap \mathscr{H}_{1, r}$, in terms of $u$ :

$$
u=\eta_{\mu}+w \quad \text { and } \quad w \perp J \mathrm{~T}_{\eta} \mathrm{M}_{\mathrm{s}} .
$$

Given Lemma 7.3 and Lemma 7.4, Proposition 7.1 follows directly.

Proof of Proposition 7.1. Lemma 7.3 states

$$
\begin{aligned}
\frac{d}{d t}\left(\mathcal{E}_{\mu}(u)+\frac{1}{2}\left\langle\mathcal{R}_{V} u, u\right\rangle\right)= & p \cdot\left\langle\nabla_{a} \mathcal{R}_{V} u, u\right\rangle-\frac{1}{2} \alpha^{\operatorname{tr}} \cdot \mathrm{D}^{2} V(a) \cdot\langle x u, u\rangle \\
& +\frac{1}{2} \dot{\mu}\|u\|_{\mathrm{L}^{2}}^{2}+\alpha^{\mathrm{b}} \cdot\langle\mathrm{i} u, \nabla u\rangle .
\end{aligned}
$$

Insert $u=\eta_{\mu}+w$ above, and use $w \perp\left\{\eta_{\mu}, \mathrm{i} \nabla \eta_{\mu}, x \eta_{\mu}\right\}$. Recall that $\eta_{\mu}$ is a real valued symmetric function, hence $\left\langle x \eta_{\mu}, \eta_{\mu}\right\rangle=0$ as well as $\left\langle\mathrm{i} \eta_{\mu}, \nabla \eta_{\mu}\right\rangle=0$. We obtain

$$
\begin{aligned}
& \frac{d}{d t}\left(\mathcal{E}_{\mu}(u)+\right.\left.\frac{1}{2}\left\langle\mathcal{R}_{V} u, u\right\rangle\right)= \\
& p \cdot\left(\left\langle\nabla_{a} \mathcal{R}_{V} w, w\right\rangle+2\left\langle\nabla_{a} \mathcal{R}_{V} \eta_{\mu}, w\right\rangle+\left\langle\nabla_{a} \mathcal{R}_{V} \eta_{\mu}, \eta_{\mu}\right\rangle\right) \\
&-\frac{1}{2} \alpha^{\mathrm{tr}} \cdot \mathrm{D}^{2} V(a) \cdot\langle x w, w\rangle+\frac{1}{2} \dot{\mu}\left(\|w\|_{\mathrm{L}^{2}}^{2}+\left\|\eta_{\mu}\right\|_{\mathrm{L}^{2}}^{2}\right)+\alpha^{\mathrm{b}} \cdot\langle\mathrm{i} w, \nabla w\rangle
\end{aligned}
$$

Subtracting the result of Lemma 7.4 we find

$$
\begin{aligned}
& \frac{d}{d t} \Lambda=p \cdot\left\langle\left(\nabla_{a} \mathcal{R}_{V}\right) w, w\right\rangle-\frac{1}{2} \alpha^{\mathrm{tr}} \cdot \mathrm{D}^{2} V(a) \cdot\langle x w, w\rangle \\
&+ \alpha^{\mathrm{b}} \cdot\langle\mathrm{i} w, \nabla w\rangle+2 p \cdot\left\langle\nabla_{a} \mathcal{R}_{V} \eta_{\mu}, w\right\rangle-\frac{1}{2} \alpha^{\mathrm{tr}} \cdot\left\langle\nabla_{a} \mathcal{R}_{V} \eta_{\mu}, \eta_{\mu}\right\rangle+\frac{\dot{\mu}}{2}\|w\|_{\mathrm{L}^{2}}^{2} \\
&-\dot{\mu}\left\langle\mathcal{R}_{V} \eta_{\mu}, \partial_{\mu} \eta\right\rangle .
\end{aligned}
$$

Note that the terms on the second and third line are at least fourth order in the small parameters. The last two lines is the definition of $R$ in the proposition. 


\section{Upper bound on $\Lambda$}

This section we estimate $\Lambda$ from above using Corollary 6.2 in Proposition 7.1. Taylor expansion of $\mathcal{E}_{\mu}(\eta(t)+w(x, t))$ around $\eta$ at $t=0$, gives

$$
\left|\mathcal{E}_{\mu(t)}(u(x, t))-\mathcal{E}_{\mu}\left(\eta_{\mu(t)}(x)\right)\right|_{t=0} \leq C\left\|w_{0}\right\|_{\mathrm{H}_{1}}^{2} .
$$

The remaining terms in the Lyapunov functional are estimated using the inequality Hess $V(x) \leq C \epsilon_{V}^{2}|x|^{2}\left\langle\epsilon_{V} x\right\rangle^{r-2}$ together with Taylor's formula and Lemma B.3. Furthermore, we use from Corollary 6.2 that $\left|\epsilon_{V} \tilde{a}_{0}\right| \leq C$. We obtain for a $\theta \in[0,1]$

$$
\begin{aligned}
\left|\left\langle\mathcal{R}_{V} u, u\right)\right\rangle-\left.\left\langle\mathcal{R}_{V} \eta, \eta\right\rangle\right|_{t=0}=\left|\left\langle\mathcal{R}_{V} w, w\right)\right\rangle+\left.2\left\langle\mathcal{R}_{V} \eta, w\right\rangle\right|_{t=0} \\
=\epsilon_{V}^{2}\left|\left\langle x \cdot \operatorname{Hess} V\left(x \theta+\tilde{a}_{0}\right) \cdot x, 2 \eta_{\mu_{0}} \operatorname{Re}\left(w_{0}\right)\right\rangle+\right|\left\langle\mathcal{R}_{V} w_{0}, w_{0}\right\rangle \mid \\
\quad \leq C\left(\epsilon_{V}^{2}\left\|w_{0}\right\|_{\mathrm{L}^{2}}+\left\|w_{0}\right\|_{\mathrm{L}^{2}}^{2}+\left\|\epsilon_{V} x\left\langle\epsilon_{V} x\right\rangle^{(r-2) / 2} w_{0}\right\|_{\mathrm{L}^{2}}^{2}\right) .
\end{aligned}
$$

We now use Corollary 5.2 and Lemma C.1 in (8.2) and (8.1) to obtain

$$
\left|\left\langle\mathcal{R}_{V} u, u\right)\right\rangle-\left.\left\langle\mathcal{R}_{V} \eta, \eta\right\rangle\right|_{t=0} \leq C\left(\epsilon_{V}^{2} \epsilon_{0}+\epsilon_{0}^{2}\right)
$$

and

Thus, finally

$$
\left|\mathcal{E}_{\mu(t)}(u(x, t))-\mathcal{E}_{\mu}\left(\eta_{\mu(t)}(x)\right)\right|_{t=0} \leq C \epsilon_{0}^{2} .
$$

$$
|\Lambda|_{t=0} \leq C\left(\epsilon_{0}^{2}+\epsilon_{V}^{2} \epsilon_{0}\right) .
$$

Proposition 8.1. Let $\psi \in U_{\delta} \cap \mathscr{H}_{1, r}$, and let $\Lambda, w$ and $\alpha$ be defined as above, and $\delta$ as defined in Theorem 4.1. Then

$$
\begin{aligned}
\left|\frac{d}{d t} \Lambda\right| \leq C\left(\left(\epsilon_{V}+\epsilon_{0}+\sqrt{\epsilon_{h}}\right) \epsilon_{V}\left\|\epsilon_{V} x\left\langle\epsilon_{V} x\right\rangle^{(r-2) / 2} w\right\|_{\mathrm{L}^{2}}^{2}+|\alpha| \epsilon_{V}\left\|\left(\epsilon_{V}|x|\right)^{1 / 2} w\right\|_{\mathrm{L}^{2}}^{2}\right. \\
\left.+\left(\left(\epsilon_{V}+\epsilon_{0}+\sqrt{\epsilon_{h}}\right) \epsilon_{V}^{2}+|\alpha|\right)\left(\|w\|_{\mathrm{H}_{1}}^{2}+\epsilon_{V}^{2}\right)\right),
\end{aligned}
$$

for times $0 \leq t \leq \min \left(T_{1}, T_{2}\right)$, where $T_{1}$ and $T_{2}$ are defined in Corollary 6.2.

Proof. Proposition 7.1 implies

$$
\begin{aligned}
\left|\frac{d}{d t} \Lambda\right| \leq & C\left(|p|\left|\left\langle\nabla_{a} \mathcal{R}_{V} w, w\right\rangle\right|+\left|\alpha^{\operatorname{tr}}\right||\operatorname{Hess} V(a) \|\langle x w, w\rangle|\right. \\
& \left.+\left|\alpha^{\mathrm{b}}\right|\|w\|_{\mathrm{L}^{2}}\|\nabla w\|_{\mathrm{L}^{2}}+|p| \epsilon_{V}^{3}\|w\|_{\mathrm{L}^{2}}+\left|\alpha^{\mathrm{tr}}\right| \epsilon_{V}^{3}+|\dot{\mu}|\|w\|_{\mathrm{L}^{2}}^{2}+|\dot{\mu}| \epsilon_{V}^{2}\right) .
\end{aligned}
$$

An alternative form of Eqn. (8.7) is

$$
\begin{aligned}
\left|\frac{d}{d t} \Lambda\right| \leq C\left(|p|\left|\left\langle\nabla_{a} \mathcal{R}_{V} w, w\right\rangle\right|+|\alpha| \mid \operatorname{Hess} V(a)\right. & \|\langle x w, w\rangle \mid \\
& \left.+\left(|p| \epsilon_{V}^{2}+|\alpha|\right)\left(\|w\|_{\mathrm{H}_{1}}^{2}+\epsilon_{V}^{2}\right)\right),
\end{aligned}
$$

where we have used $\epsilon_{V}<C$ and $\left|\alpha_{j}\right| \leq|\alpha|, \forall j$.

Using Corollary B.2 we estimate the $\mathcal{R}_{V}$ terms to obtain

$$
\begin{array}{r}
\left|\frac{d}{d t} \Lambda\right| \leq C\left(|p| \epsilon_{V}\left\|\epsilon_{V}|x|\left\langle\epsilon_{V} x\right\rangle^{(r-2) / 2} w\right\|_{\mathrm{L}^{2}}^{2}+\right. \\
+\left(|\alpha| \epsilon_{V}\left\langle\epsilon_{V}|a|_{\infty}\right\rangle^{r-2}\left|\left\langle\epsilon_{V} x w, w\right\rangle\right|\right. \\
\left.+\left(|p| \epsilon_{V}^{2}+|\alpha|\right)\left(\|w\|_{\mathrm{H}_{1}}^{2}+\epsilon_{V}^{2}\right)\right) .
\end{array}
$$


The proposition now follows upon using $\epsilon_{V}|a| \leq C_{a}$ and $|p| \leq C\left(\epsilon_{V}+\epsilon_{0}+\sqrt{\epsilon_{h}}\right)$ for $t \leq \min \left(T_{1}, T_{2}\right)$ from Corollary 6.2 and the inequality:

$$
\left\langle\epsilon_{V} x w, w\right\rangle \leq\left\|\left(\epsilon_{V}|x|\right)^{1 / 2} w\right\|_{\mathrm{L}^{2}}^{2} \text {. }
$$

Equation (8.5) and Proposition 8.1 yield an upper bound on $\Lambda$ :

$$
|\Lambda| \leq C \epsilon_{0}^{2}+C \epsilon_{V}^{2} \epsilon_{0}+t \sup _{s \leq t}\left|\frac{d}{d t} \Lambda\right| .
$$

\section{Lower bound on $\Lambda$}

In this section we estimate the Lyapunov-functional $\Lambda$ from below. Recall the definition (7.1) of $\Lambda$ :

$$
\Lambda:=\mathcal{E}_{\mu}(\eta+w)-\mathcal{E}_{\mu}(\eta)+\frac{1}{2}\left\langle\mathcal{R}_{V}(\eta+w), \eta+w\right\rangle-\frac{1}{2}\left\langle\mathcal{R}_{V} \eta, \eta\right\rangle .
$$

We have the following result.

Proposition 9.1. Let $\Lambda$ and $w$ be defined as above. Then for a positive constant $C$,

$$
\Lambda \geq \frac{1}{2} \rho_{2}\|w\|_{\mathrm{H}_{1}}^{2}+C_{0} \rho_{1}\left\|\epsilon_{V}|x|\left\langle\epsilon_{V} x\right\rangle^{(r-2) / 2} w\right\|_{\mathrm{L}^{2}}^{2}-C\|w\|_{\mathrm{H}_{1}}^{3}-C \epsilon_{V}^{2}\|w\|_{\mathrm{L}^{2}} .
$$

where $r$ and $\rho_{1}>0$ are defined in (2.4), $C_{0}$ is the positive constant defined in Lemma A.1 and $\rho_{2}>0$ is a positive number. The constant $C_{0}$ depends on the constant $C_{a}$ defined in Corollary 6.2 bounding the size of $\epsilon_{V}|a|$.

Proof. By Taylor expansion we have

$$
\mathcal{E}_{\mu}(\eta+w)-\mathcal{E}_{\mu}(\eta)=\frac{1}{2}\left\langle\mathcal{L}_{\eta} w, w\right\rangle+R_{\eta}^{(3)}(w),
$$

where $\mathcal{L}_{\eta}:=\left(\operatorname{Hess} \mathcal{E}_{\mu}\right)(\eta)$ and by Condition $2 \mathrm{a},\left|R_{\eta}^{(3)}(w)\right| \leq C\|w\|_{\mathrm{H}_{1}}^{3}$. The coercivity of $\mathcal{L}_{\eta}$ for $w \perp J \mathrm{~T}_{\eta} \mathrm{M}_{\mathrm{s}}$ is proved, under Conditions 1-3 on the nonlinearity (see Section 2), in Proposition D.1 of [17] (cf. [48]). Thus

$$
\left\langle\mathcal{L}_{\eta} w, w\right\rangle \geq \rho_{2}\|w\|_{\mathrm{H}_{1}}^{2} \text { for } w \perp J \mathrm{~T}_{\eta} \mathrm{M}_{\mathrm{s}} .
$$

The remaining terms of $\Lambda$ can be rewritten as

$$
\left\langle\mathcal{R}_{V}(\eta+w), \eta+w\right\rangle-\left\langle\mathcal{R}_{V} \eta, \eta\right\rangle=\left\langle\mathcal{R}_{V} w, w\right\rangle+2\left\langle\mathcal{R}_{V} \eta, w\right\rangle .
$$

In Lemma A.1 we show that

$$
\mathcal{R}_{V} \geq C_{0} \rho_{1}\left(\epsilon_{V}|x|\right)^{2}\left\langle\epsilon_{V} x\right\rangle^{r-2} \text { for } r \geq 1 .
$$

Using Lemma A.1, (9.4), (9.6) and the fact that $\left\langle\mathcal{R}_{V} \eta, w\right\rangle \leq C \epsilon_{V}^{2}\|w\|_{\mathrm{L}^{2}}$ we obtain the lower bound on $\Lambda$. 


\section{Proof of Theorem 2.1}

The upper bound (8.11) together with the bound from below in Proposition 9.1 yield the inequality

$$
\begin{gathered}
\frac{1}{2} \rho_{2}\|w\|_{\mathrm{H}_{1}}^{2}+C_{0} \rho_{1}\left\|\epsilon_{V} x\left\langle\epsilon_{V} x\right\rangle^{(r-2) / 2} w\right\|_{\mathrm{L}^{2}}^{2}-C\|w\|_{\mathrm{H}_{1}}^{3}-C \epsilon_{V}^{2}\|w\|_{\mathrm{L}^{2}} \leq C \epsilon_{0}^{2}+C \epsilon_{V}^{2} \epsilon_{0} \\
+t C \sup _{s \leq t}\left(\left(\epsilon+\sqrt{\epsilon_{h}}\right) \epsilon_{V}\left\|\epsilon_{V} x\left\langle\epsilon_{V} x\right\rangle^{(r-2) / 2} w\right\|_{\mathrm{L}^{2}}^{2}+|\alpha| \epsilon_{V}\left\|\left(\epsilon_{V}|x|\right)^{1 / 2} w\right\|_{\mathrm{L}^{2}}^{2}\right. \\
\left.+\left(\left(\epsilon+\sqrt{\epsilon_{h}}\right) \epsilon_{V}^{2}+|\alpha|\right)\left(\|w\|_{\mathrm{H}_{1}}^{2}+\epsilon_{V}^{2}\right)\right), \quad(10.1)
\end{gathered}
$$

for $0 \leq t \leq \min \left(T_{1}, T_{2}\right)$, where $T_{1}$ and $T_{2}$ are defined in Corollary 6.2 and $\epsilon:=$ $\epsilon_{V}+\epsilon_{0}$. The right-hand side is independent of the operator $t \mapsto s, \sup _{s<t}$ in the given time interval, we can therefore apply this to both sides of (10.1). To simplify, let

$$
\rho:=\min \left(\frac{\rho_{2}}{8}, \frac{C_{0} \rho_{1}}{3}\right) .
$$

We absorb higher order terms into lower order ones. Furthermore, we assume

$$
t \leq \min \left(T_{1}, T_{2}, T_{3}\right), \quad \text { where } T_{3}:=\frac{\rho}{C\left(|\alpha|_{\infty}+\epsilon_{V}\left(\epsilon+\sqrt{\epsilon_{h}}\right)\right)\left(1+\epsilon_{V}\right)},
$$

in agreement with Corollary 6.2. Both $\rho$ and $C$ above depend on $I$, clarifying the need for $\epsilon \ll C(I)$. Note that

$$
T_{3} C\left(\epsilon+\sqrt{\epsilon_{h}}\right) \epsilon_{V} \leq \rho, T_{3} C|\alpha|_{\infty} \epsilon_{V} \leq \rho, \text { and } T_{3} C\left(\left(\epsilon+\sqrt{\epsilon_{h}}\right) \epsilon_{V}^{2}+|\alpha|_{\infty} \leq 2 \rho .\right.
$$

We obtain

$$
\begin{gathered}
\rho \sup _{s \leq t}\left(4\|w\|_{\mathrm{H}_{1}}^{2}+3\left\|\epsilon_{V} x\left\langle\epsilon_{V} x\right\rangle^{(r-2) / 2} w\right\|_{\mathrm{L}^{2}}^{2}\right) \\
\quad \leq C\left(\sup _{s \leq t}\left(\|w\|_{\mathrm{H}_{1}}^{3}+\epsilon_{V}^{2}\|w\|_{\mathrm{L}^{2}}\right)+\epsilon_{0}^{2}+\epsilon_{V}^{2} \epsilon_{0}\right) \\
+\rho \sup _{s \leq t}\left(\left\|\epsilon_{V} x\left\langle\epsilon_{V} x\right\rangle^{(r-2) / 2} w\right\|_{\mathrm{L}^{2}}^{2}+\left\|\left|\epsilon_{V} x\right|^{1 / 2} w\right\|_{\mathrm{L}^{2}}^{2}+2 \epsilon_{V}^{2}+2\|w\|_{\mathrm{H}_{1}}^{2}\right) .
\end{gathered}
$$

Note that $g(y):=|y|-y^{2}\langle y\rangle^{-1} \leq 2^{-1}, y \in \mathbb{R}$. Indeed $g(-y)=g(y)$ and $g$ is continuously differentiable on $(0, \infty), g(y) \geq 0$ since $|y| \geq y^{2}\langle y\rangle^{-1}$ with $g(0)=$ $g(\infty)=0$. The function $g(y)$ has one critical point on $(0, \infty)$ at $y=\left(2^{-1}(\sqrt{5}-\right.$ $1))^{-1 / 2}$ with value $\max g=(3-\sqrt{5})(2(\sqrt{5}-1))^{-1 / 2} \leq 2^{-1}$. This proves the claim. We now use this intermediate function $g(x)$ to estimate the term above with $|x|^{1 / 2}$. We have

$$
\epsilon_{V}|x|-\left(\epsilon_{V}|x|\right)^{2}\left\langle\epsilon_{V} x\right\rangle^{r-2} \leq g\left(\epsilon_{V}|x|\right) \leq \frac{1}{2} .
$$

We also have the inequalities

$$
C\|w\|_{\mathrm{H}_{1}}^{3} \leq \rho^{-1} C^{2}\|w\|_{\mathrm{H}_{1}}^{4}+4^{-1} \rho\|w\|_{\mathrm{H}_{1}}^{2}, \quad C \epsilon_{V}^{2}\|w\|_{\mathrm{H}_{1}} \leq C^{2} \rho^{-1} \epsilon_{V}^{4}+4^{-1} \rho\|w\|_{\mathrm{H}_{1}}^{2} .
$$


Thus we have $3 \rho\|w\|_{\mathrm{H}_{1}}^{2}$ on the right-hand side and $2 \rho$ of terms containing $\left\langle\epsilon_{V} x\right\rangle$. Moving those to the left-hand side of (10.5) using the above inequalities and simplifying we obtain

$$
\sup _{s \leq t}\left(\|w\|_{\mathrm{H}_{1}}^{2}+\left\|\epsilon_{V} x\left\langle\epsilon_{V} x\right\rangle^{(r-2) / 2} w\right\|_{\mathrm{L}^{2}}^{2}\right) \leq C^{\prime} \epsilon^{2}+C^{2} \rho^{-2}\left(\sup _{s \leq t}\|w\|_{\mathrm{H}_{1}}^{4}\right) .
$$

Abbreviate $\kappa:=C^{\prime} \epsilon^{2}$. Let

$$
X:=\sup _{s \leq t}\left(\|w\|_{\mathrm{H}_{1}}^{2}+\left\|\epsilon_{V}|x|\left\langle\epsilon_{V} x\right\rangle \epsilon_{V} x^{(r-2) / 2} w\right\|_{\mathrm{L}^{2}}^{2}\right) .
$$

Equation (10.8) implies

$$
X \leq C^{2} \rho^{-2} X^{2}+\kappa .
$$

Solving this inequality, we find

$$
X \leq 2 \kappa, \quad \text { provided } \kappa \leq \frac{\rho^{2}}{4 C^{2}} .
$$

The definition of $X$ and $\kappa$ implies

$$
\|w\|_{\mathrm{H}_{1}} \leq c^{\prime} \epsilon, \quad \text { and } \quad\left\|\epsilon_{V} x\left\langle\epsilon_{V} x\right\rangle \epsilon_{V} x^{(r-2) / 2} w\right\|_{\mathrm{L}^{2}} \leq c^{\prime} \epsilon
$$

Lemma C.1 allow us to rewrite (10.12) as $\|w\|_{\mathscr{H}_{1, r}} \leq c^{\prime} \epsilon$. Inserting (10.12) into the expressions for our modulation parameters, the estimate of the $\alpha_{j}$-terms in (4.25)-(4.28) gives us $|\alpha| \leq c \epsilon^{2}$ and time interval $t \leq T^{\prime}$, where

$$
T^{\prime}:=c \min \left(\epsilon^{-2}, \frac{\sqrt{\epsilon_{h}}}{\epsilon^{2}}, \frac{1}{\epsilon^{2}+\epsilon_{V} \sqrt{\epsilon_{h}}}\right) .
$$

Using $\epsilon_{h} \geq K \epsilon_{V}$ (that is, $\epsilon_{h}$ is not an order of magnitude smaller then $\epsilon_{V}$ ), we can shorten the time-interval to have an upper limit of

$$
T^{\prime \prime}:=C\left(\epsilon^{2}+\epsilon_{V} \sqrt{\epsilon_{h}}\right)^{-1} .
$$

We now choose $\epsilon$ such that (10.11) holds and $c^{\prime} \epsilon \leq \frac{1}{2} \delta$, where $\delta$ is defined in Theorem 4.1. Then there is a maximum $T_{0}$ such that the solution $\psi$ of (1.1) is in $U_{\delta}$ for $t \leq T_{0}$. Thus the decomposition (4.4) is valid and the above upper bounds for $\|w\|_{\mathrm{H}_{1}}$ and $\alpha$ are valid for $t \leq \min \left(T_{0}, C\left(\epsilon^{2}+\epsilon_{V} \sqrt{\epsilon_{h}}\right)^{-1}\right)$. Thus there exists a constant $C_{T}$ such that $0<C_{T} \leq C$, such that for $t \leq C_{T}\left(\epsilon^{2}+\epsilon_{V} \sqrt{\epsilon_{h}}\right)^{-1}$ the theorem holds. This concludes the proof of Theorem 2.1 .

\section{Appendix A. Lower bound on $\mathcal{R}_{V}$}

In this appendix we estimate $\mathcal{R}_{V}$ from below. Recall that

$$
\mathcal{R}_{V}(x):=V(x+a)-V(a)-\nabla V(a) \cdot x
$$

and

$$
\text { Hess } V \geq \rho_{1} \epsilon_{V}^{2}\left\langle\epsilon_{V} x\right\rangle^{(r-2) / 2},
$$

where $\rho_{1}$ is a positive constant. We have the following result: 
Lemma A.1. Let $a, x \in \mathbb{R}^{d}$ and $0<\epsilon_{V} \in \mathbb{R}$. Then (i) if $r \geq 2$ or (ii) if $r \leq 2$ and $\epsilon_{V}|a| \leq C_{a}$ :

$$
\mathcal{R}_{V}(x) \geq C_{0} \rho_{1} \epsilon_{V}^{2}|x|^{2}\left\langle\epsilon_{V} x\right\rangle^{r-2},
$$

where

$$
C_{0}:= \begin{cases}\frac{1}{2^{r-2+\max \left(0, \frac{r-4}{2}\right)} r(r-1)} & \text { in case }(\mathrm{i}) \\ \frac{1}{2\left(2\left(1+C_{a}^{2}\right)\right)^{(2-r) / 2}} & \text { in case }(\mathrm{ii}) .\end{cases}
$$

Proof. Consider the case $x=0$, from the definition of $\mathcal{R}_{V}$ it follows that $\mathcal{R}_{V}(0)$ $=0$, thus the estimate holds. Let $x \neq 0$, the function $\mathcal{R}_{V}(x)$ is the Taylor expansion of $V(x+a)$ around $a$ to first order. The Taylor series remainder

$$
\int_{0}^{1}(1-\theta) x \cdot \operatorname{Hess} V(a+\theta x) \cdot x \mathrm{~d} \theta,
$$

integrated by parts, gives the identity

$$
\begin{aligned}
& \int_{0}^{1}(1-\theta) x \cdot \operatorname{Hess} V(a+\theta x) \cdot x \mathrm{~d} \theta= \\
& \left.(1-\theta) x \cdot \nabla V(a+\theta x)\right|_{0} ^{1}+\int_{0}^{1} \nabla V(a+\theta x) \cdot x \mathrm{~d} \theta=\mathcal{R}_{V}(x, t) .
\end{aligned}
$$

Inserting the lower bound of the Hess $V$ into the Taylor remainder gives the inequality

$$
\mathcal{R}_{V}(x) \geq \rho_{1} \epsilon_{V}^{2}|x|^{2} \int_{0}^{1}(1-\theta)\left(1+\epsilon_{V}^{2}|a+x \theta|^{2}\right)^{(r-2) / 2} \mathrm{~d} \theta=\rho_{1} \epsilon_{V}^{2}|x|^{2} I .
$$

To estimate $I$, we first consider case (a), with $r \geq 2$. The integrand of $I$ is estimated by the following lemma.

Lemma A.2. Let $y \geq 0$ and $b \geq 0$ then

$$
\frac{1}{2^{\max \left(0, \frac{2-b}{2}\right)}} \leq \frac{\left(1+y^{2}\right)^{b / 2}}{1+|y|^{b}} \leq 2^{\max \left(0, \frac{b-2}{2}\right)} .
$$

This lemma is proved at the end of this appendix. For $x \neq 0$ denote $\hat{x}=x /|x|$, $a_{\|}=a \cdot \hat{x}$, and $a_{\perp}=a-a_{\|} \hat{x}$, and abbreviate $b:=r-2$. Lemma A.2 then implies

$$
\begin{aligned}
\left(1+\epsilon_{V}^{2}|a+x \theta|^{2}\right)^{b / 2} & =\left(1+\epsilon_{V}^{2}\left|a_{\perp}\right|^{2}\right)^{b / 2}\left(1+\frac{\epsilon_{V}^{2}}{1+\epsilon_{V}^{2}\left|a_{\perp}\right|^{2}}\left(a_{\|}+|x| \theta\right)^{2}\right)^{b / 2} \\
& \geq \frac{1}{2^{\max \left(0, \frac{2-b}{2}\right)}}\left(\left(1+\epsilon_{V}^{2}\left|a_{\perp}\right|^{2}\right)^{b / 2}+\epsilon_{V}^{b}\left|a_{\|}+\right| x|\theta|^{b}\right) \\
& \geq \frac{1}{2}+\frac{1}{2} \epsilon_{V}^{b}\left|a_{\|}+\right| x|\theta|^{b}
\end{aligned}
$$

where we used $2^{\max \left(0, \frac{2-b}{2}\right)} \leq 2$. Thus

$$
I \geq \frac{1}{4}+\frac{1}{2} \epsilon_{V}^{r-2} \int_{0}^{1}(1-\theta)\left|a_{\|}+\right| x|\theta|^{r-2} \mathrm{~d} \theta=\frac{1}{4}+\frac{1}{2} \epsilon_{V}^{r-2} I_{2} .
$$


The integral $I_{2}$ evaluates to

$$
I_{2}=\frac{1}{r(r-1)|x|^{2}}\left(\left|a_{\|}+\right| x||^{r}-\left|a_{\|}\right|^{r}-r|x| \operatorname{sign}\left(a_{\|}\right)\left|a_{\|}\right|^{r-1}\right),
$$

which is the remainder of a Taylor series of $\left|a_{\|}+\right| x||^{r}$ around $a_{\|}$, as expected. At $a_{\|}=0$ we have

$$
I_{2} \geq \frac{|x|^{r-2}}{r(r-1)} .
$$

To estimate $I_{2}$ for $a_{\|} \neq 0$, we use the following lemma

Lemma A.3. Let $y \in \mathbb{R}$ and $r \geq 2$. Then

$$
|1+y|^{r}-1-r y \geq \frac{1}{2^{r-2}}|y|^{r} .
$$

The lemma is proved at the end of this appendix. Since $a_{\|} \neq 0$ we can pull it out of $I_{2}$, and use $y:=|x| / a_{\|}$in the lemma to obtain

$$
I_{2} \geq \frac{|x|^{r-2}}{2^{r-2} r(r-1)}
$$

Note that the above result is a lower limit than we obtained in (A.12), so we can use (A.14) for all $a_{\|}$. Now, inserting this inequality into (A.10) and the result into (A.7) to obtain

$$
\mathcal{R}_{V}(x) \geq \rho_{1} \epsilon_{V}^{2}|x|^{2} I \geq \rho_{1}\left(\frac{\epsilon_{V}^{2}|x|^{2}}{4}+\frac{\epsilon_{V}^{r}|x|^{r}}{2^{r-1} r(r-1)}\right) .
$$

Once again using Lemma A.2 gives

$$
\mathcal{R}_{V}(x) \geq C_{1} \rho_{1} \epsilon_{V}^{2}|x|^{2}\left\langle\epsilon_{V} x\right\rangle^{r-2},
$$

where

$$
C_{1}:=\frac{1}{2^{\max \left(0, \frac{r-4}{2}\right)} 2^{r-2} r(r-1)}, \quad r \geq 2 .
$$

This concludes part (i) of Lemma A.1, except for the proofs of Lemma A.2 and A.3 which is done below.

Now, we estimate the integral $I$ for the case (ii), with $r \leq 2$ and $\epsilon_{V}|a| \leq C_{a}$. Introduce the change of variables $p=(1-\theta)^{2}$. The integral takes the form

$$
I=\frac{1}{2} \int_{0}^{1} \frac{1}{g(p)^{(2-r) / 2}} \mathrm{~d} p, \quad \text { where } g(p):=1+\epsilon_{V}^{2}|a+x(1+\sqrt{p})|^{2} .
$$

The triangle inequality together with $0 \leq p \leq 1$ gives

$$
g(p) \leq 1+2 \epsilon_{V}^{2}|a|^{2}+2 \epsilon_{V}^{2}|x|^{2}(1-\sqrt{p}) \leq\left(1+\max \left(2 \epsilon_{V}^{2}|a|^{2}, 1\right)\right)\left\langle\epsilon_{V} x\right\rangle^{2} .
$$

The upper bound $\epsilon_{V}|a| \leq C_{a}$ and the estimate $1+\max \left(2 \epsilon_{V}^{2}|a|^{2}, 1\right) \leq 2+2 C_{a}^{2}$ together with a trivial integral gives that $I$ is bounded from below as

$$
I \geq C_{2}\left\langle\epsilon_{V} x\right\rangle^{r-2}, \quad \text { where } C_{2}:=\frac{1}{2\left(2\left(1+C_{a}^{2}\right)\right)^{(2-r) / 2}} .
$$

Inserting this result into (A.7) concludes the lemma. 
Now consider Lemma A.2. It is a combination of the inequalities Theorem 13 and Theorem 19 in [24].

Lemma A.4. Let $y \in \mathbb{R}$ and $b \geq 0$ then

$$
\frac{1}{2^{\max \left(0, \frac{2-b}{2}\right)}} \leq \frac{\left(1+y^{2}\right)^{b / 2}}{1+|y|^{b}} \leq 2^{\max \left(0, \frac{b-2}{2}\right)} .
$$

Proof. Denote

$$
f(y, b):=\frac{\left(1+y^{2}\right)^{b / 2}}{1+|y|^{b}} .
$$

We first note that $f(y, b)=f(-y, b)$, thus we can restrict our attention to $y \geq 0$. At $y=0$ we have $f(0, b)=1$ and at $y=\infty, f(\infty, b)=1$. The function is differentiable for $y>0$, the only critical point for $y>0$ is at $y=1$, where the function takes the value

$$
f(1, b)=2^{b / 2-1} .
$$

If $b>2$ its a maximum, and if $b<2$ its a minimum, the lemma follows.

To prove Lemma A.3 we begin by stating it again.

Lemma. Let $y \in \mathbb{R}$ and $r \geq 2$. Then

$$
|1+y|^{r}-1-r y \geq \frac{1}{2^{r-2}}|y|^{r} .
$$

Proof. Denote

$$
f(y ; r):=|1+y|^{r}-1-r y-\frac{1}{2^{r-2}}|y|^{r} .
$$

The lemma is equivalent to $f \geq 0$, for $r \geq 2$. We note that $f$ is twice differentiable at all points except $y=-1$ and $y=0$. We observe that the inequality is satisfied for both of these points since we have $f(0 ; r)=0$ and $f(-1, r)=r-1-\frac{1}{2^{r-1}}>0$ for $r \geq 2$. Consider the derivative of $f$ with respect to $y$ :

$$
\partial_{y} f(y ; r)=r\left(\operatorname{sign}(1+y)|1+y|^{r-1}-\frac{1}{2^{r-2}} \operatorname{sign}(y)|y|^{r-1}-1\right) .
$$

We wish to show that $f$ decays monotonically on $y<0$ and hence, that $\partial_{y} f \leq 0$ for $-1<y<0$ and $y<-1$. We also wish to show that $f$ increases monotonically for $y>0$ with $\partial_{y} f \geq 0$. To show this, consider first the case $y>0$ : using that $(1+y)^{r-1} \geq 1+y^{r-1}$, we have $\partial_{y} f>0$ for $y>0$.

For the interval $-1<y<0$, use that $b^{r}<b$ for any $b \in(0,1)$, to obtain

$$
\begin{aligned}
\partial_{y} f(y ; r)=(1-|y|)^{r-1}+2^{2-r}|y|^{r-1}-1 & <-|y|+2^{2-r}|y| \\
& =-\left(1-2^{2-r}\right)|y| \leq 0, \quad r \geq 2 .
\end{aligned}
$$

For the last interval $y<-1$ we re-write $($ A.26) as

$$
\partial_{y} f(y ; r)=-r\left((|y|-1)^{r-1}-2^{2-r}|y|^{r-1}+1\right) .
$$

Upon calculating $\partial_{y}^{2} f$, and solving $\partial_{y}^{2} f=0$ for $y$ in this interval we find that $\partial_{y} f$ has a maximum at $|y|=2$ with value $\partial_{y} f(2 ; r)=0$. Hence, $\partial_{y} f \leq 0$ for $y \leq-1$ 
and $-1<y<0$. We have showed that $f$ decays monotonically on $y<0$ and increases monotonically on $y>0$, and $f(0 ; r)=0$. Hence $f \geq 0$ for all $y \in \mathbb{R}$, which proves the lemma.

\section{Appendix B. Upper bound on $\mathcal{R}_{V}$ and $\nabla_{a} \mathcal{R}_{V}$}

In this appendix we estimate $\mathcal{R}_{V}$ and derivatives of $\mathcal{R}_{V}$ from above. From the proof of Lemma A.1 we have the following identity

$$
\mathcal{R}_{V}(x)=\int_{0}^{1}(1-\theta) x \cdot \operatorname{Hess} V(a+\theta x) \cdot x \mathrm{~d} \theta .
$$

Furthermore, in (2.4) we made the assumptions that, for $\beta \in \mathbb{Z}^{d}$ and $|\beta| \leq 3$,

$$
\left|\partial_{x}^{\beta} V(x)\right| \leq C_{V} \epsilon_{V}^{|\beta|}\left\langle\epsilon_{V} x\right\rangle^{r-|\beta|} .
$$

We begin with the following result for derivatives of $\mathcal{R}_{V}$.

Lemma B.1. Let $a, x \in \mathbb{R}^{d}, 0<\epsilon_{V} \in \mathbb{R}$ and $\epsilon_{V}|a| \leq C_{a}$, as in Corollary 6.2. Furthermore let $\beta \in \mathbb{Z}^{d}$, with $0 \leq \beta_{j} \leq 1 \forall j=1, \ldots, d$ and $|\beta|=1$. Then, (i) if $r \geq 2$ :

$$
\left|\partial_{a}^{\beta} \mathcal{R}_{V}\right| \leq C_{1} \epsilon_{V}^{3}|x|^{2}\left\langle\epsilon_{V} x\right\rangle^{\max (r-3,0)},
$$

or (ii) if $1 \leq r<2$ :

where

$$
\left|\partial_{a}^{\beta} \mathcal{R}_{V}\right| \leq C_{2} \epsilon_{V}^{3}|x|^{2}\left\langle\epsilon_{V} x\right\rangle^{r-2}
$$

$$
C_{1}:=2^{-1} C_{V} d\left(2\left(1+C_{a}^{2}\right)\right)^{\max (r-3,0) / 2}, \quad C_{2}:=C_{V} d\left(6 \sqrt{2}+\ln \left(1+C_{a}\right)\right) .
$$

Here $C_{V}$ is the constant in (2.4).

Corollary B.2. Under the same conditions as in Lemma B.1 we have

$$
\left|\partial_{a}^{\beta} \mathcal{R}_{V}\right| \leq C \epsilon_{V}^{3}|x|^{2}\left\langle\epsilon_{V} x\right\rangle^{r-2},
$$

where $C$ depends on $C_{1}$ and $C_{2}$ above.

Proof. Use that $\left\langle\epsilon_{V} x\right\rangle^{\max (r-3,0)} \leq\left\langle\epsilon_{V} x\right\rangle^{r-2}$ in Lemma B.1.

Proof of Lemma B.1. For the case $x=0,\left(\partial_{a}^{\beta} \mathcal{R}_{V}\right)(0)=0$ and thus the lemma is satisfied. For $x \neq 0$, and since $V \in \mathrm{C}^{3}$, we have from (B.1) that

$$
\partial_{a}^{\beta} \mathcal{R}_{V}(x, t)=\sum_{k, l=1}^{d} x_{l} x_{k} \int_{0}^{1}(1-\theta)\left(\partial_{x}^{\beta} \partial_{x_{k}} \partial_{x_{l}} V\right)(a+\theta x) \mathrm{d} \theta .
$$

The upper bound on the potential, (B.2), gives

$$
\left|\partial_{a_{j}} \mathcal{R}_{V}(x)\right| \leq \epsilon_{V}^{3} C_{V} \sum_{k, l=1}^{d}\left|x_{l} x_{k}\right| \int_{0}^{1}(1-\theta)\left(1+\epsilon_{V}^{2}|a+\theta x|^{2}\right)^{(r-3) / 2} \mathrm{~d} \theta,
$$


here $C_{V}$ is the constant in (2.4). To estimate $\left|\partial_{a}^{\beta} \mathcal{R}_{V}\right|$, we use the inequality

$$
\sum_{k, l=1}^{d}\left|x_{k} x_{l}\right| \leq d|x|^{2}
$$

to obtain

$$
\left|\partial_{a}^{\beta} \mathcal{R}_{V}(x)\right| \leq C_{V} d \epsilon_{V}^{3}|x|^{2} \int_{0}^{1}(1-\theta)\left(1+\epsilon_{V}^{2}|a+\theta x|^{2}\right)^{(r-3) / 2}=d C_{V} \epsilon_{V}^{3}|x|^{2} I .
$$

To estimate the integrand, we consider first case (i), with $r>3, \epsilon_{V}|a| \leq C_{a}$. Before we estimate the integral $I$, we estimate part of its integrand with the triangle inequality together with $\epsilon_{V}|a| \leq C_{a}$ and $\theta \leq 1$ to obtain

$$
1+\epsilon_{V}^{2}|a+\theta x|^{2} \leq 1+2 C_{a}^{2}+2|x|^{2} \leq\left(1+\max \left(2 C_{a}^{2}, 1\right)\right)\left\langle\epsilon_{V} x\right\rangle^{2} .
$$

Thus

$$
\left|\partial_{a}^{\beta} \mathcal{R}_{V}(x, t)\right| \leq \tilde{C}_{1} \epsilon_{V}^{3}|x|^{2}\left\langle\epsilon_{V} x\right\rangle^{r-3}
$$

where

$$
\tilde{C}_{1}:=2^{-1} C_{V} d\left(2\left(1+C_{a}^{2}\right)\right)^{(r-3) / 2} .
$$

To extend this case to include $r \geq 2$, we note that for $r \in[2,3]$ the exponent in the integrand of I, $r-3 \leq 0$, and that $1+\epsilon_{V}^{2}|a+\theta x|^{2} \geq 1$ to obtain

$$
I \leq 2^{-1} \text {. }
$$

We conclude that for $r \in[2,3]\left|\partial_{a}^{\beta} \mathcal{R}_{V}\right| \leq 2^{-1} d C_{V} \epsilon_{V}^{3}|x|^{2}$. Thus upon changing (B.12) and (B.13) into

$$
\left|\partial_{a}^{\beta} \mathcal{R}_{V}(x)\right| \leq C_{1} \epsilon_{V}^{3}|x|^{2}\left\langle\epsilon_{V} x\right\rangle^{\max (r-3,0)}
$$

where

$$
C_{1}:=2^{-1} C_{V} d\left(2\left(1+C_{a}^{2}\right)\right)^{\max (r-3,0) / 2},
$$

part (i) is proved.

For the case (ii), with $r<2$ we need a more precise estimate that the case of $r \in[2,3]$. To obtain this, recall that $x \neq 0$ and let us introduce the notations $\hat{x}:=x /|x|, a_{\|}:=a \cdot \hat{x}$ and $a_{\perp}:=a-a_{\|}$. Then $|a+\theta x|^{2}=a_{\perp}^{2}+\left|a_{\|}+\theta\right| x||^{2}$. By the change of variables $y=\epsilon_{V}\left(a_{\|}+\theta|x|\right)$ and that $1+a_{\perp}^{2} \geq 1$, we find

$$
I \leq \frac{1}{\epsilon_{V}|x|} \int_{\epsilon_{V} a_{\|}}^{\epsilon_{V}\left(a_{\|}+|x|\right)} \frac{\mathrm{d} y}{\left(1+y^{2}\right)^{(3-r) / 2}}=: \frac{1}{\epsilon_{V}|x|} I_{2} .
$$

The goal is to show that $\left\langle\epsilon_{V} x\right\rangle^{2-r} I$ is bounded by a constant independent of $\epsilon_{V}$. To show this, we consider two intervals for $|x|$ first $\epsilon_{V}|x| \leq 1$. For this interval $\left\langle\epsilon_{V} x\right\rangle^{2-r} \leq 2^{2-r}$ and $I_{2} \leq \epsilon_{V}|x|$, thus $\left\langle\epsilon_{V} x\right\rangle^{2-r} I \leq 2$.

For the intervals $\epsilon_{V}|x| \geq 1$ and $1 \leq r \leq 1.5$. We show that $I_{2}$ is bounded by a constant. Indeed, regardless of the values of $a_{\|}$and $|x|$ we have

$$
I_{2} \leq 2 \int_{0}^{\infty} \frac{\mathrm{d} y}{\left(1+y^{2}\right)^{(3-r) / 2}}=\sqrt{\pi} \frac{\Gamma\left(1-\frac{r}{2}\right)}{\Gamma\left(\frac{r-3}{2}\right)} \leq 6
$$


for $1 \leq r \leq 1.5$. Thus

$$
\left\langle\epsilon_{V} x\right\rangle^{2-r} I \leq 6 \frac{\left\langle\epsilon_{V} x\right\rangle^{2-r}}{\epsilon_{V}|x|} \leq 6 \sqrt{2} .
$$

For $r \in[1.5,2]$ we need a better estimate, we use that $\left(1+y^{2}\right)^{(3-r) / 2} \geq$ $\left(1+y^{2}\right)^{1 / 2}$, thus

$$
I_{2} \leq \int_{\epsilon_{V} a_{\|}}^{\epsilon_{V}\left(a_{\|}+|x|\right)} \frac{\mathrm{d} y}{\sqrt{1+y^{2}}}=\ln \left(\frac{\epsilon_{V} a_{\|}+\epsilon_{V}|x|+\sqrt{1+\epsilon_{V}^{2}|a+x|^{2}}}{\epsilon_{V} a_{\|}+\sqrt{1+\epsilon_{V}^{2} a_{\|}^{2}}}\right) .
$$

To estimate this, we consider four different regions. For $a_{\|}>0$ and $|x|>|a|$ it is bounded by $\ln \left(1+4 \epsilon_{V}|x|\right)$. For $a_{\|}>0$ and $|x| \leq|a|$ it is bounded by $\ln 2$. For $a_{\|}<0$ and $|x|<|a|$ it is bounded by $\ln \left(1+2 C_{a}\right)$. For $a_{\|}<0$ and $|x|>|a|$ it is bounded by $\ln \left(\left(1+2 C_{a}\right)\left(1+4 \epsilon_{V}|x|\right)\right)$. Thus

$$
\begin{aligned}
I_{2} & \leq \ln \left(2+2 C_{a}\right)+\ln \left(1+4 \epsilon_{V}|x|\right) \\
& \leq\left(\epsilon_{V}|x|\right)^{1 / 2} \ln \left(10\left(1+C_{a}\right)\right), \quad \text { for } \epsilon_{V}|x| \geq 1,
\end{aligned}
$$

where we have used that for $\epsilon_{V}|x| \geq 1, q \geq 0$ we have $q+\ln \left(1+4 \epsilon_{V}|x|\right) \leq$ $(q+\ln 5)\left(\epsilon_{V}|x|\right)^{1 / 2}$. Thus

$$
\left\langle\epsilon_{V} x\right\rangle^{2-r} I \leq \ln \left(10\left(1+C_{a}\right)\right) \frac{\left\langle\epsilon_{V} x\right\rangle^{2-r}}{\left(\epsilon_{V}|x|\right)^{1 / 2}} \leq 2^{1 / 4} \ln \left(10\left(1+C_{a}\right)\right) .
$$

Comparing the constants above for the $I$ estimate we find that

$$
C_{2}:=C_{V} d\left(6 \sqrt{2}+\ln \left(1+C_{a}\right)\right),
$$

is sufficient. This concludes the proof of the lemma.

To bound $\mathcal{R}_{V}$ from above we could use the same methods as above, but the upper bound will be to large to fit into the energy space. But we have the following

Lemma B.3. For $r \geq 1$ and $\epsilon_{V}|a| \leq C_{a}$

$$
\mathcal{R}_{V} \leq C_{1}\left(1+\epsilon_{V}^{2}|x|^{2}\left\langle\epsilon_{V} x\right\rangle^{r-2}\right),
$$

where

$$
C_{1}:=2 C_{V}\left(2+2 C_{a}^{2}\right)^{(r-1) / 2} .
$$

Proof. For $r \geq 2$, we there exists a $\theta \in[0,1]$ such that

$$
\mathcal{R}_{V} \leq C_{V} \epsilon_{V}^{2}|x|^{2}\langle x \theta+a\rangle^{r-2} .
$$

Since $r-2 \geq 0$ we estimate $\epsilon_{V}|x \theta+a| \leq \epsilon_{V}|x|+C_{a}$ and $\left(1+2 a|x|^{2}+2 C_{a}^{2}\right) \leq$ $\left(2+2 C_{a}^{2}\right)\left\langle\epsilon_{V} x\right\rangle^{2}$ we obtain the lemma for $r \geq 2$ as

$$
\mathcal{R}_{V} \leq C_{V}\left(2+2 C_{a}^{2}\right)^{(r-2) / 2}\left|\epsilon_{V}\right|^{2}|x|^{2}\left\langle\epsilon_{V} x\right\rangle^{r-2} .
$$

For $r \in[1,2)$ we use that there exists a $\theta \in[0,1]$ such that

$$
\mathcal{R}_{V}=(\nabla V(x \theta+a, t)-\nabla V(a)) \cdot x \leq C_{V} \epsilon_{V}|x|\left(\left\langle\epsilon_{V}(x \theta+a)\right\rangle^{r-1}+\left\langle C_{a}\right\rangle^{r-1}\right) .
$$


Once again $\epsilon_{V}|x \theta+a| \leq \epsilon_{V}|x|+C_{a}$ and we obtain

$$
\mathcal{R}_{V} \leq C_{V} \epsilon_{V}|x|\left(\left(2+2 C_{a}^{2}\right)^{(r-1) / 2}\left\langle\epsilon_{V} x\right\rangle^{r-1}+\left\langle C_{a}\right\rangle^{r-1}\right) .
$$

To estimate the second term, recall (10.6), to get

$$
\epsilon_{V}|x| \leq 2^{-1}+\left(\epsilon_{V}|x|\right)^{2}\left\langle\epsilon_{V} x\right\rangle^{r-2} .
$$

To estimate the first term in (B.29), let $y=\epsilon_{V}|x| \geq 0, r \in[1,2]$ and calculate

$$
y\langle y\rangle^{r-1}-y^{2}\langle y\rangle^{r-2}=y\langle y\rangle^{r-1}\left(1-\frac{y}{\langle y\rangle}\right)=\frac{y\langle y\rangle^{r-1}}{(y+\langle y\rangle)\langle y\rangle} \leq \frac{y}{(y+\langle y\rangle)} \leq \frac{1}{2} .
$$

Thus

$$
\epsilon_{V}|x|\left\langle\epsilon_{V} x\right\rangle^{r-1} \leq 2^{-1}+\left(\epsilon_{V}|x|\right)^{2}\left\langle\epsilon_{V} x\right\rangle^{r-2} .
$$

Collecting the above two terms gives

$$
\mathcal{R}_{V} \leq 2 C_{V}\left(2+2 C_{a}^{2}\right)^{(r-1) / 2}\left(1+\left(\epsilon_{V}|x|\right)^{2}\left\langle\epsilon_{V} x\right\rangle^{r-2}\right) .
$$

Since (B.33) for $r \geq 2$ is larger than (B.27) we have proved the lemma.

\section{Appendix C. Bound in energy-space}

In Eq. (10.12) we show that

$$
\|w\|_{0}:=\|w\|_{\mathrm{H}_{1}}+\left\|\epsilon_{V}|x|\left\langle\epsilon_{V} x\right\rangle^{(r-2) / 2} w\right\|_{\mathrm{L}^{2}} \leq C \epsilon .
$$

We want to show that $\|w\|_{\mathscr{H}_{1, r}} \leq C_{r}\|w\|_{0}$. This result follows from the following lemma:

Lemma C.1. For $r \in(0, \infty)$ there exists a constant $c_{r}$ such that

$$
0<1+\min \left(0, c_{r}\right) \leq \frac{1+y^{2}\langle y\rangle^{r-2}}{\langle y\rangle^{r}} \leq 1+\max \left(0, c_{r}\right)<2,
$$

where

$$
c_{r}:=\frac{2-r}{2}\left(\frac{2}{r}\right)^{2 /(r-2)}, \quad r \neq 2 \text {, }
$$

and for $r=2, c_{r}=0$.

Proof. Denote

$$
f(z)=\frac{1+\left(z^{2}-1\right) z^{r-2}}{z^{r}}=1+z^{-r}-z^{-2}, \quad z \geq 1 .
$$

Note that for $z^{2}=1+y^{2}, f$ is the function we want to estimate for the lemma. For $r=2, f=1$, thus $c_{r}=0$. The function $f$ is at least $\mathrm{C}^{1}$ for $z \geq 1$. Now consider $r \neq 2$. We note that $f(1)=1$ and $f \rightarrow 1$ as $z \rightarrow \infty$. The critical point on $[1, \infty)$ of $f$ is at $z_{c}:=(r / 2)^{1 /(r-2)}>1$, where the function take the value

$$
f\left(z_{c}\right)=1+\frac{2-r}{r}\left(\frac{2}{r}\right)^{2 /(r-2)} .
$$

A maximum (minimum) for $r<2(r>2)$. This concludes the proof. 


\section{Appendix D. A family of time-dependent solutions}

In this appendix, we construct a family of solutions to the nonlinear Schrödinger equation with a quadratic, time-independent potential.

Let $\psi(x, t)$ have the form

$$
\psi(x, t)=\mathrm{e}^{\mathrm{i} p(t) \cdot(x-a(t))+\mathrm{i} \gamma(t)} \tilde{\eta}_{\mu}(x-a(t)),
$$

where $\tilde{\eta}_{\mu}$ is a real-valued function, not yet determined. We substitute this function into (1.1), and let $y:=x-a$ to obtain

$$
0=\dot{p} \cdot y \tilde{\eta}_{\mu}+\left(\dot{\gamma}+p^{2}-\dot{a} \cdot p\right) \tilde{\eta}_{\mu}+\mathrm{i} \nabla \tilde{\eta}_{\mu} \cdot(\dot{a}-2 p)-\Delta \tilde{\eta}_{\mu}-f\left(\tilde{\eta}_{\mu}\right)+V(y+a) \tilde{\eta}_{\mu}
$$

By adding and subtracting the terms $(\mu+V(a)) \tilde{\eta}_{\mu}$ and $\nabla V(a) \cdot y \tilde{\eta}_{\mu}$ and as usual defining $\mathcal{R}_{V}:=V(y+a)-V(a)-\nabla V(a) \cdot y$ we find

$$
\begin{aligned}
0=(\dot{p}+\nabla V(a)) \cdot y \tilde{\eta}_{\mu}+\left(\dot{\gamma}+p^{2}-\dot{a} \cdot p\right. & +V(a)-\mu) \tilde{\eta}_{\mu}+\mathrm{i} \nabla \tilde{\eta}_{\mu} \cdot(\dot{a}-2 p) \\
& +(-\Delta+\mu) \tilde{\eta}_{\mu}-f\left(\tilde{\eta}_{\mu}\right)+\mathcal{R}_{V} \tilde{\eta}_{\mu} .
\end{aligned}
$$

If we choose

$$
\dot{p}=-\nabla V(a), \quad \dot{a}=2 p, \quad \dot{\gamma}=p^{2}+\mu-V(a),
$$

then the Eqn. D.3 reduces to

$$
0=-\Delta \tilde{\eta}_{\mu}+\mu \tilde{\eta}_{\mu}-f\left(\tilde{\eta}_{\mu}\right)+\mathcal{R}_{V} \tilde{\eta}_{\mu}
$$

where $\tilde{\eta}_{\mu}=\tilde{\eta}_{\mu}(y)$, and $\Delta=\sum \partial_{y_{j}}^{2}$. In general this equation is time-dependent due to the appearance of $a$ in $\mathcal{R}_{V}$, but for potentials of the form $V(x)=x \cdot A \cdot x+v \cdot x+d$, with constant matrix $A$, vector $v$ and scalar $d$, we have

$$
\begin{aligned}
\mathcal{R}_{V}=(y+a) \cdot A \cdot(y+a)+v & (y+a)+d-(a \cdot A \cdot a+v \cdot a+d) \\
& -(a \cdot A \cdot y+y \cdot A \cdot a+v \cdot y)=y \cdot A \cdot y .
\end{aligned}
$$

The right-hand side is independent of $a$, and hence of time. Equation (D.3) reduces to

$$
0=-\Delta \tilde{\eta}_{\mu}+\mu \tilde{\eta}_{\mu}-f\left(\tilde{\eta}_{\mu}\right)+y \cdot A \cdot y \tilde{\eta}_{\mu} .
$$

Thus, if there exists nontrivial solutions to this equation, we have constructed a family of solutions $\mathrm{e}^{\mathrm{i} p(t) \cdot(x-a)+\mathrm{i} \gamma} \tilde{\eta}_{\mu}(x-a)$, where $\tilde{\eta}_{\mu}$ solves (D.7). Existence of solutions to a general class of equations that includes (D.7) under some restrictions on $b:=\mu+y \cdot A \cdot y$ and with a class of local nonlinearities is shown by Rabinowitz [34] and extended to more general potentials by Sikarov [36]. Sikarov require the following potential conditions: $b>-c$, where $|c|<\infty$,

$$
\inf _{u \in \mathrm{H}_{1},\|u\|_{\mathrm{L}^{2}}=1}\|\nabla u\|_{\mathrm{L}^{2}}^{2}+\langle b u, u\rangle>0
$$

and that $b$ grows to infinity in almost all directions as $|y| \rightarrow \infty$.

Acknowledgment. Part of this work was done while one of the authors (IMS) was visiting ETH Zürich and ESI Vienna. IMS is grateful to J. Fröhlich and to P.C. Aichelburg and P. Bizoń for their hospitality. The authors are grateful to the 
referee for useful remarks and for providing several relevant references originally missed by the authors.

\section{References}

[1] H. Berestycki and P.-L. Lions, Nonlinear scalar field equations. I. Existence of a ground state, Arch. Rational Mech. Anal. 82 (4) (1983), 313-345, doi:10.1007/ BF00250555.

[2] H. Berestycki and P.-L. Lions, Nonlinear scalar field equations. II. Existence of infinitely many solutions, Arch. Rational Mech. Anal. 82 (4) (1983), 347-375, doi:10.1007/ BF00250556.

[3] H. Berestycki, P.-L. Lions, and L.A. Peletier, An ODE approach to the existence of positive solutions for semilinear problems in $\mathbb{R}^{N}$, Indiana Univ. Math. J. 30 (1) (1981), 141-157, doi:10.1512/iumj.1981.30.30012.

[4] J.C. Bronski and R.L. Jerrard, Soliton dynamics in a potential, Math. Res. Lett. 7 (2-3) (2000), 329-342, ams:MR1764326.

[5] V.S. Buslaev and G.S. Perel'man, Scattering for the nonlinear Schrödinger equation: states that are close to a soliton, Algebra i Analiz 4 (6) (1992), 63-102, ams: MR1199635.

[6] V.S. Buslaev and G.S. Perel'man, On the stability of solitary waves for nonlinear Schrödinger equations, Amer. Math. Soc. Transl. Ser. 2 (164) (1995), 74-98, ams: MR1334139.

[7] V.S. Buslaev and C. Sulem, On asymptotic stability of solitary waves for nonlinear Schrödinger equations, Ann. I.H.P. Analyse Nonlinéaire 20 (2003), 419-475, doi: 10.1016/S0294-1449(02)00018-5.

[8] R. Carles, Semi-classical Schrödinger equations with harmonic potential and nonlinear perturbation, Ann. I.H.P., Analyse non linéaire 20 (3) (2003), 501-542, doi: 10.1016/S0294-1449(02)00027-6.

[9] T. Cazenave, An introduction to nonlinear Schrödinger equations. Number 26 in Textos de Métodos Matemáticos. Instituto de Matematica - UFRJ, Rio de Janeiro, RJ, third edition, 1996.

[10] S. Cuccagna, Stabilization of solutions to nonlinear Schrödinger equations, Comm. Pure Appl. Math. 54 (9) (2001), 1110-1145, doi:10.1002/cpa.1018.

[11] S. Cuccagna, Erratum: "Stabilization of solutions to nonlinear Schrödinger equations", Comm. Pure Appl. Math. 58 (1) (2005), 147, doi:10.1002/cpa.20052.

[12] S. Cuccagna, On asymptotic stability of ground states of NLS, Rev. Math. Phys. 15 (8) (2003), 877-903, doi:10.1142/S0129055X03001849.

[13] S.I. Dejak and B.L.G. Jonsson, Long-time dynamics of variable coefficient $m K d V$ solitary waves, submitted, 2005, ArXiv: math-ph/0503016.

[14] S.I. Dejak and I.M. Sigal, Long-time dynamics of KdV solitary waves over a variable bottom, Comm. Pure Appl. Math. (2006), doi: 10.1002/cpa.20120. Published online.

[15] G. Derks and E. van Groesen, Energy propagation in dissipative systems. Part II: Centrovelocity for nonlinear wave equations, Wave Motion 15 (1992), 159-172. doi: 10.1016/0165-2125(92)90016-U. 
[16] P. Ehrenfest, Bemerkung über die angenäherte Gültigkeit der klassischen Mechanik innerhalb der Quantenmechanik (German) [Note on the approximate validity of classical mechanics in quantum mechanics], Zeitschrift für Physik 45 (7-8) (1927), 455457, doi: 10.1007/BF01329203.

[17] J. Fröhlich, S. Gustafson, B.L.G. Jonsson, and I.M. Sigal, Solitary wave dynamics in an external potential, Comm. Math. Phys. 250 (3) (2004), 613-642, doi:10.1007/ s00220-004-1128-1.

[18] J. Fröhlich, T.-P. Tsai, and H.-T. Yau, On a classical limit of quantum theory and the non-linear Hartree equation, Geom. Funct. Anal., Special Volume, Part I (2000), 57-78, ams:MR1826249.

[19] J. Fröhlich, T.-P. Tsai, and H.-T. Yau, On the point-particle (Newtonian) limit of the non-linear Hartree equation, Comm. Math. Phys. 225 (2) (2002), 223-274, doi: $10.1007 / \mathrm{s} 002200100579$.

[20] Z. Gang and I.M. Sigal, Asymptotic stability of nonlinear Schrödinger equations with potential, Rev. Math. Phys 17 (10) (2005), 1143-1207, doi:10.1142/ S0129055X05002522.

[21] M. Grillakis, J. Shatah, and W. Strauss, Stability theory of solitary waves in the presence of symmetry. II, J. Funct. Anal. 94 (2) (1990), 308-348, doi: 10.1016/00221236(90)90016-E.

[22] E. van Groesen and F. Mainardi, Energy propagation in dissipative systems. Part I: Centrovelocity for linear systems, Wave Motion 11 (1989) 201-209, doi:10.1016/01652125(89)90001-2.

[23] S. Gustafson, K. Nakanishi, and T.-P. Tsai, Asymptotic stability and completeness in the energy space for nonlinear Schrödinger equations with small solitary waves, Int. Math. Res. Not. 66 (2004), 3559-3584, doi:10.1155/S1073792804132340.

[24] G.H. Hardy, J.E. Littlewood, and G. Pólya, Inequalities. Cambridge University Press, second edition, 1967.

[25] D.J. Kaup, A perturbation expansion for the Zakharov-Shabat inverse scattering transform, SIAM J. Appl. Math. 31 (1) (1976), 121-133, doi:10.1137/0131013.

[26] S. Keraani, Semiclassical limit of a class of Schrödinger equations with potential, Comm. PDE. 27 (3 \& 4) (2002), 693-704, doi:10.1081/PDE-120002870.

[27] Y. Kodama and M.J. Ablowitz, Perturbations of solitons and solitary waves, Stud. Appl. Math. 64 (3) (1981) 225-245, ams:MR615541.

[28] E. Lenzmann, Well-posedness for Hartree NLS with electromagnetic field of arbitrary growth, In preparation, 2006.

[29] D.W. McLaughlin and A.C. Scott, Pertubation analysis of fluxon dynamics, Phys. Rev. A 18 (4) (1978) 1652-1680, doi:10.1103/PhysRevA.18.1652.

[30] K. McLeod, Uniqueness of positive radial solutions of $\Delta u+f(u)=0$ in $\mathbb{R}^{n}$, II, Am. Math. Soc. 339 (2) (1993), 495-505, ams:MR1201323.

[31] F. Merle and P. Raphael, The blow-up dynamic and upper bound on the blow-up rate for critical nonlinear Schrödinger equation, Ann. of Math. (2) 161 (1) (2005), 157-222, ams:MR2150386.

[32] Y.-G. Oh, Cauchy problem and Ehrenfest's law of nonlinear Schrödinger equations with potentials, J. Differential Equations 81 (2) (1989), 255-274, doi:10.1016/00220396(89)90123-X. 
[33] G. Perelman, Asymptotic stability of multi-soliton solutions for nonlinear Schrödinger equations, Comm. PDE 29 (7-8) (2004), 1051-1095, doi:10.1081/PDE-200033754.

[34] P.H. Rabinowitz, On a class of nonlinear Schrödinger equations, Z. Angew. Math. Phys. 43 (2) (1992), 270-291, doi:10.1007/BF00946631.

[35] I. Rodnianski, W. Schlag, and A. Soffer, Asymptotic stability of $n$-soliton states of NLS, (2003), ArXiv:math.AP/0309114.

[36] B. Sirakov, Existence and multiplicity of solutions of semi-linear elliptic equations in $\mathbb{R}^{n}$, Calc. Var. 11 (2) (2000), 119-142, doi: 10.1007/s005260000010.

[37] A. Soffer and M.I. Weinstein, Multichannel nonlinear scattering theory for nonintegrable equations. In Integrable systems and applications (Île d'Oléron, 1988), volume 342 of Lecture Notes in Phys., pages 312-327. Springer, Berlin, 1989, ams: MR1034551.

[38] A. Soffer and M.I. Weinstein, Multichannel nonlinear scattering for nonintegrable equations, Comm. Math. Phys. 133 (1) (1990) 119-146, doi:10.1007/BF02096557.

[39] A. Soffer and M.I. Weinstein, Multichannel nonlinear scattering for nonintegrable equations II. The case of anisotropic potentials and data, J. Differential Equations 98 (2) (1992), 376-390, doi:10.1016/0022-0396(92)90098-8.

[40] A. Soffer and M.I. Weinstein, Selection of the ground state for nonlinear Schrödinger equations, Reviews in Math. Phys. 16(8) (2004), 977-1071, doi:10.1142/ S0129055X04002175.

[41] D.M.A. Stuart, Perturbation theory for kinks, Comm. Math. Phys. 149 (3) (1992), 433-462, ams:MR1186038.

[42] D.M.A. Stuart, Modulational approach to stability of non-topological solitons in semilinear wave equations, J. Math. Pures Appl. (9) 80 (1) (2001), 51-83, doi:10.1016/ S0021-7824(00)01189-2.

[43] D.M.A. Stuart, The geodesic hypothesis and non-topological solitons on pseudo-Riemannian manifolds, Ann. Sci. École Norm. Sup. (4) 37 (2) (2004), 312-362, doi: 10.1016/j.ansens.2003.07.001.

[44] D.M.A. Stuart, Geodesics and the Einstein nonlinear wave system, J. Math. Pures Appl. (9) 83 (5) (2004), 541-587, doi:10.1016/j.matpur.2003.09.009.

[45] T.-P. Tsai and H.-T. Yau, Asymptotic dynamics of nonlinear Schrödinger equations: resonance-dominated and dispersion-dominated solutions, Comm. Pure Appl. Math. 55 (2) (2002), 153-216, doi:10.1002/cpa.3012.

[46] T.-P. Tsai and H.-T. Yau, Relaxation of excited states in nonlinear Schrödinger equations, Int. Math. Res. Not. 31 (2002), 1629-1673, doi:10.1155/S1073792802201063.

[47] T.-P. Tsai and H.-T. Yau, Stable directions for excited states of nonlinear Schrödinger equations, Comm. PDE 27 (11-12) (2002), 2363-2402, doi:10.1081/PDE-120016161.

[48] M.I. Weinstein, Modulational stability of ground states of nonlinear Schrödinger equations, SIAM J. Math. Anal. 16 (3) (1985), 472-491, ams:MR0783974.

[49] M.I. Weinstein, Lyapunov stability of ground states of nonlinear dispersive evolution equations, Comm. Pure Appl. Math. 39 (1) (1986), 51-68, doi:10.1137/0516034. 
[50] K. Yajima and G. Zhang, Smoothing property for Schrödinger equations with potential superquadratic at infinity, Comm. Math. Phys. 221 (3) (2001), 573-590, doi: 10.1007/s002200100483.

[51] K. Yajima and G. Zhang, Local smoothing property and Strichartz inequality for Schrödinger equations with potentials superquadratic at infinity, J. Differential Equations 202 (1) (2004), 81-110, doi:10.1016/j.jde.2004.03.027.

B. Lars G. Jonsson

Institute für Theoretische Physik

ETH Zürich

CH-8093 Zürich

Switzerland

and

Electrical Engineering

Royal Institute of Technology

SE-100 44 Stockholm

Sweden

e-mail: jonsson@phys.ethz.ch

Jürg Fröhlich

Institute für Theoretische Physik

ETH Zürich

CH-8093 Zürich

Switzerland

e-mail: juerg@itp.phys.ethz.ch

Stephen Gustafson

Department of Mathematics

University of British Columbia

Vancouver, BC, V6T 1Z2

Canada

e-mail: gustaf@math .ubc . ca

Israel Michael Sigal

Department of Mathematics

University of Toronto

Toronto, ON, M5S 2E4

Canada

e-mail: im.sigal@utoronto.ca

Communicated by Rafael D. Benguria

Submitted: March 7, 2005

Accepted: January 9, 2006

(10) To access this journal online:

[4P) http://www.birkhauser.ch 